\title{
Abundance of attracting, repelling and elliptic periodic orbits in two-dimensional reversible maps.
}

\author{
Delshams A. $\dagger$, Gonchenko S.V.‡, Gonchenko V.S.‡, Lázaro \\ J.T.† and Sten'kin O. $\ddagger$ \\ $\dagger$ Universitat Politècnica de Catalunya, Barcelona, Spain \\ $\ddagger$ Institute for Applied Mathematics \& Cybernetics, N.Novgorod, Russia
}

\begin{abstract}
We study dynamics and bifurcations of two-dimensional reversible maps having non-transversal heteroclinic cycles containing symmetric saddle fixed points. We consider one-parameter families of reversible maps unfolding the initial heteroclinic tangency and prove the existence of infinitely many sequences (cascades) of bifurcations and birth of asymptotically stable, unstable and elliptic periodic orbits.
\end{abstract}

Dedicated to the memory of Leonid Pavlovich Shilnikov, a Master whose works strongly influenced the mathematical theory of dynamical systems.

\section{Introduction}

Reversible systems have a very special status inside the realm of dynamical systems. They are often positioned "between" dissipative and conservative systems. For continuous dynamical systems, reversibility means that the system is invariant under the change in time-direction, $t \mapsto-t$, and a transformation in the spatial variables. In the discrete context, reversibility of a map $f$ (a diffeomorphism) means that $f$ and $f^{-1}$ possess the "same" dynamics, where the term "same" can have rather different meanings. For instance, if $f$ and $f^{-1}$ are smoothly conjugate, that is, $f \circ h=h \circ f^{-1}$ where $h$ is a general diffeomorphism, then $f$ is called weekly reversible. Much more interesting types of reversibility appear when $h$ possesses some structures. For example, if $h$ is an involution, i.e. $h^{2}=\mathrm{Id}$ (and $h \neq \mathrm{Id}$ ), then the map $f$ is called strongly reversible. Since this case is the most frequent in the literature, nowadays strongly reversible maps are simply named reversible maps.

In contrast to conservative and dissipative systems, the study of homoclinic bifurcations in reversible systems is not so popular. Even for two-dimensional maps, only few results are known and most of them relate to "conservative and reversible" maps which form a certain codimension- $\infty$ subclass in the class of reversible maps. This situation is probably due to a "common belief" that conservative and dissipative phenomena of dynamics exist separately and, thus, there is no clear necessity to 
study them "all together". However, they can actually appear in this way. Indeed, a dynamical system exhibits the so-called phenomenon of mixed dynamics when:

(i) it has simultaneously infinitely many hyperbolic periodic orbits of all possible types (stable, completely unstable and saddle), and

(ii) these orbits are not separated as a whole, that is, the closures of sets of orbits of different types have non-empty intersections.

The property of mixed dynamics seems to be a generic property, i.e., it holds for residual subsets of open regions of systems. This fact is almost straightforward consequence of the Newhouse work [17], taking into account also results of GavrilovShilnikov and Newhouse $[6,18]$ on bifurcations of homoclinic tangencies: it is sufficiently to consider tangencies associated to thick horseshoes containing saddles with Jacobians bigger and less than one. However, it is absolutely not clear where such thick horseshoes exist. In principle, it does not matter, since it was proved in [10] that such regions, Newhouse regions with mixed dynamics, exist near any two-dimensional diffeomorphism with a non-transversal heteroclinic cycle containing at least two saddle periodic points $O_{1}$ and $O_{2}$ satisfying that $\left|J\left(O_{1}\right)\right|>1$ and $\left|J\left(O_{2}\right)\right|<1$, where $J\left(O_{i}\right)$ is the Jacobian of the Poincaré map at the point $O_{i}, i=1,2$.

Let us recall that a heteroclinic cycle (contour) is a set consisting of (ordered) saddle hyperbolic periodic orbits $O_{1}, \ldots, O_{n}$, as well as several heteroclinic orbits $\Gamma_{i, j} \subset W^{u}\left(O_{i}\right) \cap W^{s}\left(O_{j}\right)$ containing, at least, the orbits $\Gamma_{i, i+1}$, for $i=1, \ldots, n-1$, and $\Gamma_{n, 1}$. In general, cycles can include also homoclinic orbits $\Gamma_{i, i} \subset W^{u}\left(O_{i}\right) \cap W^{s}\left(O_{i}\right)$. An heteroclinic cycle is called non-transversal (or non-rough) if at least one of the intersections $W^{u}\left(O_{i}\right) \cap W^{s}\left(O_{j}\right)$ is non-transversal.

Remind also that Newhouse regions are open (in $C^{2}$-topology) domains in the space of dynamical systems in which systems with homoclinic tangencies are dense. It is known that they exist in any neighbourhood of any system having a homoclinic tangency to a saddle periodic orbit $[19,9]$. Such regions appear in parameter families unfolding, generally, the initial homoclinic tangency. In the parameter space, the values of the parameters corresponding to the existence of homoclinic tangencies are dense in open domains (see, for instance, [19, 9, 20, 24] and [5] for area-preserving maps), called Newhouse intervals in the case of one-parameter families.

The existence of Newhouse regions near systems with non-transversal heteroclinic cycles follows from these works. Moreover, as it was proved in [10], Newhouse intervals with heteroclinic tangencies (of the same type as the initial one) exist in any general one-parameter unfolding.

It is worth mentioning that contracting-expanding heteroclinic cycles are rather usual among reversible maps, as shown in Figure 1(a). In that example the reversible map has two saddle fixed points $O_{1}$ and $O_{2}$ and two heteroclinic orbits $\Gamma_{12} \subset W^{u}\left(O_{1}\right) \cap W^{s}\left(O_{2}\right)$ and $\Gamma_{21} \subset W^{u}\left(O_{2}\right) \cap W^{s}\left(O_{1}\right)$ such that $R\left(O_{1}\right)=O_{2}$ and $R\left(\Gamma_{21}\right)=\Gamma_{21}, R\left(\Gamma_{12}\right)=\Gamma_{12}$. Besides, the orbit $\Gamma_{12}$ is non-transversal, so that the manifolds $W^{u}\left(O_{1}\right)$ and $W^{s}\left(O_{2}\right)$ have a quadratic tangency along $\Gamma_{12}$. Since $R\left(O_{1}\right)=O_{2}$ it turns out that $J\left(O_{1}\right)=J^{-1}\left(O_{2}\right)$. If $J\left(O_{i}\right) \neq \pm 1, i=1,2$, then the heteroclinic cycle is contracting-expanding. This condition is robust and is perfectly compatible with reversibility.

Certainly, results of [10] can be applied to reversible maps with such heteroclinic cycles. However, reversible systems are sharply different from general ones by the fact that they can possess robust non-hyperbolic symmetric periodic orbits, e.g. elliptic symmetric periodic points. This leads to the idea that the phenomenon of mixed 
dynamics in the case of two-dimensional reversible maps should be connected with the coexistence of infinitely many attracting, repelling, saddle and elliptic periodic orbits. It appears to be true that the phenomenon of mixed dynamics is universal

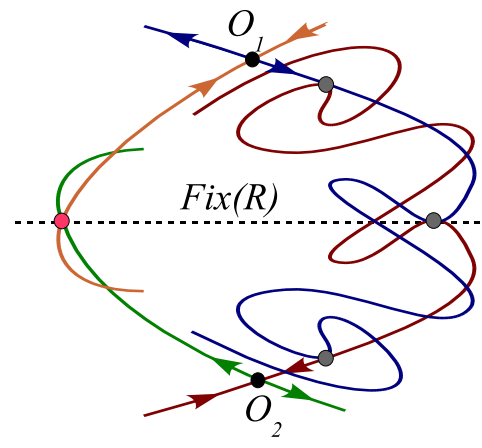

(a)

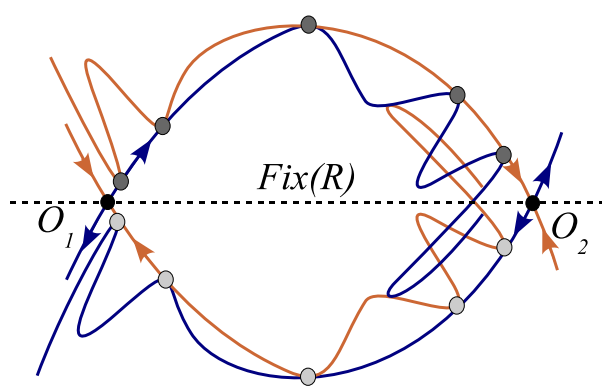

(b)

Figure 1. Two examples of planar reversible maps with symmetric nontransversal (quadratic tangency) heteroclinic cycles: (a) of the first type (there is a non-transversal symmetric heteroclinic orbit to a symmetric couple of saddle points, and (b) of the second type - there is a symmetric couple of non-transversal heteroclinic orbits to symmetric saddle points.

for reversible (two-dimensional) maps with complicated dynamics when symmetric structures (symmetric periodic, homoclinic and heteroclinic orbits) are involved. This universality can be formulated as follows:

Reversible Mixed Dynamics Conjecture Two-dimensional reversible maps with mixed dynamics are generic in Newhouse regions where maps with symmetric homoclinic or/and heteroclinic tangencies are dense.

We will assume, from now on, that the involution $R$ is not trivial and leaves fixed a curve, that is, it satisfies

$$
R^{2}=\mathrm{Id}, \quad \operatorname{dim} \text { Fix } R=1,
$$

where Fix $R=\left\{(x, y) \in \mathbb{R}^{2} \mid R(x, y)=(x, y)\right\}$. We say that an object $\Lambda$ is symmetric when $R(\Lambda)=\Lambda$. To put more emphasis, the notation self-symmetric may be used. By a symmetric couple of objects $\Lambda_{1}, \Lambda_{2}$, we mean two different objects which are symmetric one to each other, i.e., $R\left(\Lambda_{1}\right)=\Lambda_{2}$. Symmetric homoclinic (heteroclinic) tangencies can be divided into two main types, namely, when: (i) there is a nontransversal symmetric heteroclinic orbit to a symmetric couple of saddle points, or (ii) there is a symmetric homoclinic tangency or a symmetric couple of non-transversal homo/heteroclinic orbits to symmetric saddle points.

The heteroclinic quadratic tangency shown in Fig. 1(a) relates to the first type. This case was studied in the paper of Lamb and Stenkin [15] where, in fact, the RMD-Conjecture was proved for general one-parameter reversible unfoldings, under the generic condition $J\left(O_{1}\right)=J^{-1}\left(O_{2}\right) \neq 1$.

Our main goal in this paper is to prove the RMD-Conjecture for general oneparameter reversible unfoldings of heteroclinic tangencies of the second type. Indeed, 
let us consider a one-parameter family $\left\{f_{\mu}\right\}$ of $R$-reversible maps having, for $\mu=0$, a map with a symmetric non-transversal heteroclinic cycle as the one in Fig. 1(b). That is, $f_{0}$ has two symmetric saddle fixed points $O_{1}$ and $O_{2}$ and a symmetric couple of heteroclinic orbits $\Gamma_{12} \subset W^{u}\left(O_{1}\right) \cap W^{s}\left(O_{2}\right)$ and $\Gamma_{21} \subset W^{u}\left(O_{2}\right) \cap W^{s}\left(O_{1}\right)$, where $R\left(O_{1}\right)=O_{1}, R\left(O_{2}\right)=O_{2}$ and $R\left(\Gamma_{12}\right)=\Gamma_{21}$. Since $O_{1}, O_{2} \in$ Fix $R$, the condition $J\left(O_{1}\right)=J\left(O_{2}\right)=1$ holds always. Therefore, our genericity condition, the condition [C] from Section 2, relates to nonconservative properties of the map near any heteroclinic orbit $\Gamma_{12}$ or $\Gamma_{21}$.
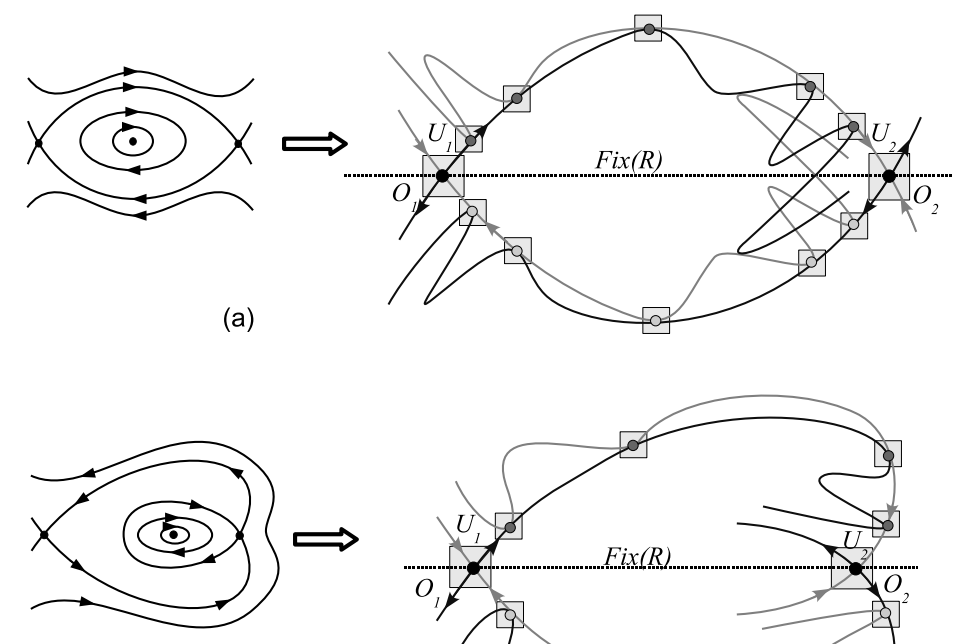

(b)

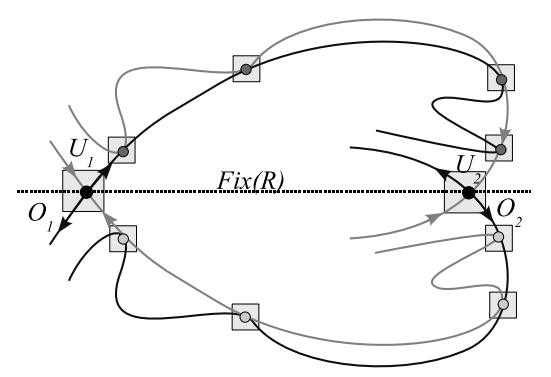

Figure 2. Examples of reversible maps with non-transversal heteroclinic cycles in the cases of (a) "inner tangency"; (b) "outer tangency". These diffeomorphisms can be constructed as the Poincaré maps for periodically perturbed conservative planar systems (left) when the corresponding perturbations preserve the reversibility (for example, with respect to the change $x \rightarrow x, y \rightarrow-y, t \rightarrow-t$, as in the system $\left.\dot{x}=y, \dot{y}=x-x^{3}+\varepsilon \dot{x} \cos t\right)$. Notice that the resulting reversible map cannot be area-preserving, in general

In the family $\left\{f_{\mu}\right\}$ we study bifurcations of single round periodic orbits, that is, orbits which pass just one time along a small fixed neighbourhood $U$ of the contour $O_{1} \cup O_{2} \cup \Gamma_{12} \cup \Gamma_{21}$. For such orbits we construct the first return (Poincaré) maps and study bifurcations of their fixed points. In Theorem 1 it is stated that infinitely many (cascade) bifurcations of birth and symmetry-breaking of single-round periodic orbits occur when varying $\mu$ near 0 . The birth bifurcation is a nondegenerate reversible "fold bifurcation" under which a pair of $R$-symmetric saddle and elliptic fixed points appear in the corresponding first return map. One of these points undergoes next a nondegenerate reversible "pitch-fork bifurcation" which leads to the appearance of a couple of contracting-expanding fixed points. The type of this bifurcation depends on a character of the heteroclinic tangency: (a) "inner tangency" and (b) "outer tangency" (see Fig. 2). In the case of "inner tangency", the elliptic fixed point falls into saddle, sink and source; in the case of "outer tangency", otherwise, the saddle 
fixed point falls into elliptic and two saddles with Jacobians $>1$ and $<1$. Some illustration of these bifurcations is shown in Fig. 3. Let us remark that the cascade of bifurcations found is different from the well-known cascades of bifurcations observed in one-parameter unfoldings of quadratic homoclinic tangencies for general systems. In the latter case we have, first of all, the cascade of bifurcations "saddle-node plus first period doubling" , [6], which, as was posed by Shilnikov in [26] and proved by Alligood and Yorke in [32], is only the first step of the cascade of bifurcations "saddle-node plus infinite sequences of period doublings".

The main result of this paper, contained in Theorem 2, is the existence of Newhouse intervals with mixed dynamics, which is almost an immediate consequence of Theorem 1. Both theorems are stated in Section 2. Section 3 is devoted to the proof of Theorem 1.

Section 4 contains two concrete examples of ordinary differential equations where the results in Section 2 can be applied to their associated Poincaré maps. First, in Subsection 4.1, it is shown that reversible two-dimensional maps with a priori nonconservative orbit behavior can be obtained as certain periodic perturbations of twodimensional reversible conservative flows. As a concrete example we consider Duffing equation. In this way we can obtain a realization of a reversible map of the second type, as in Figure 2. Subsection 4.2 is devoted to the Pikovsky-Topaj model [21] of three coupled rotators in the 3 -dimensional torus which gives rise to a reversible map on the two-dimensional torus exhibiting a reversible non-conservative orbit behavior.

In Section 5, we consider the so-called cross-form representation of reversible twodimensional maps. It is well-known that such type of cross-forms of maps are very convenient for studying hyperbolic properties of systems with homoclinic orbits, both transversal [25] and non-transversal [6, 14]. Since L.P. Shilnikov was the first author introducing such forms and coordinates, they are often referred to as "Shilnikov crossform" and "Shilnikov coordinates". This cross-form appear to be pretty convenient to deal with reversible maps. And last (but not least), Section 6 contains the proofs of the lemmas needed in the proof of Theorem 1.

\section{Symmetry breaking bifurcations in the case of reversible maps with non-transversal heteroclinic cycles}

Let $f_{0}$ be a $C^{r}$-smooth, $r \geq 4$, two-dimensional map, reversible with respect to an involution $R$ satisfying $\operatorname{dim} \operatorname{Fix}(R)=1$. Let us assume that $f_{0}$ satisfies the following two conditions:

[A] $f_{0}$ has two saddle fixed points $O_{1}$ and $O_{2}$ belonging to the line $\operatorname{Fix}(R)$ and that any point $O_{i}$ has multipliers $\lambda_{i}, \lambda_{i}^{-1}$ with $0<\lambda_{i}<1, i=1,2$.

[B] The invariant manifolds $W^{u}\left(O_{1}\right)$ and $W^{s}\left(O_{2}\right)$ have quadratic tangencies at the points of some heteroclinic orbit $\Gamma_{12}$ and, therefore, by reversibility, the manifolds $W^{u}\left(O_{2}\right)$ and $W^{s}\left(O_{1}\right)$ have quadratic tangencies at the points of a heteroclinic orbit $\Gamma_{21}=R\left(\Gamma_{12}\right)$.

Hypotheses $[A]-[B]$ define reversible maps with non-transversal symmetric heteroclinic cycles like in Figure 1(b). We ask them to satisfy one more condition. Namely, consider two points $M_{1} \in W_{l o c}^{u}\left(O_{1}\right)$ and $M_{2} \in W_{l o c}^{s}\left(O_{2}\right)$ belonging to the same heteroclinic orbit $\Gamma_{12}$ and suppose $f_{0}^{q}\left(M_{1}\right)=M_{2}$ for a suitable integer $q$. Let some smooth local coordinates $\left(x_{i}, y_{i}\right)$ be chosen near the points $O_{i}$ in such a way that the local invariant manifolds are straightened, i.e., $W_{l o c}^{u}\left(O_{i}\right)$ and $W_{l o c}^{s}\left(O_{i}\right)$ have, respectively, equations 
$x_{i}=0$ and $y_{i}=0$. Let $T_{12}$ denote the restriction of the map $f_{0}^{q}$ onto a small neighbourhood of the point $M_{1}$. Then, we assume that

[C] the Jacobian of $T_{12}$ is not constant and, moreover,

$$
Q=\left.\frac{\partial J\left(T_{12}\right)}{\partial y}\right|_{M_{1}} \neq 0
$$

Condition $J\left(T_{12}\right) \neq$ constant is well defined only when certain restrictions on the local coordinates hold. One possibility is when these coordinates $\left(x_{i}, y_{i}\right)$ around $O_{i}$ are chosen in such a way that $W_{l o c}^{u}\left(O_{i}\right)$ and $W_{l o c}^{s}\left(O_{i}\right)$ are locally straightened, i.e. have equations $\left\{x_{i}=0\right\}$ and $\left\{y_{i}=0\right\}$, respectively. However, the sign of $J\left(T_{12}\right)$ depends also on the orientation chosen for the coordinate axes. To be precise, we choose these orientations in such a a way that the heteroclinic points $M_{1} \in W_{\text {loc }}^{u}\left(O_{1}\right), M_{1}^{\prime}=$ $R\left(M_{1}\right) \in W_{l o c}^{s}\left(O_{1}\right), M_{2}=T_{12}\left(M_{1}\right) \in W_{l o c}^{s}\left(O_{2}\right)$ and $M_{2}^{\prime}=R\left(M_{2}\right) \in W_{l o c}^{u}\left(O_{2}\right)$ have positive their coordinates $y_{1}, x_{1}, x_{2}$ and $y_{2}$, respectively (see Fig. 4 , where one can put $\left.M_{1}=M_{1}^{-}, M_{2}=M_{2}^{+}, M_{1}^{\prime}=M_{1}^{+}, M_{2}^{\prime}=M_{2}^{-}\right)$.

The generic condition [C] implies that the (global) map defined near a heteroclinic point is neither a linear contraction (expansion) nor a conservative map. $\ddagger$

Two classes of reversible maps satisfying conditions [A]-[C] can be distinguished: those maps with "inner" (heteroclinic) tangency and those with "outer" tangency, corresponding to $J\left(T_{12}\right)>0$ and $J\left(T_{12}\right)<0$, respectively. Two examples of such diffeomorphisms are shown in Figure 2. Notice that in both cases the global map $T_{12}$ preserves orientation. In the case (a) the axes $x_{1}, y_{1}$ and $x_{2}, y_{2}$ have the same orientation, whereas the orientations are different in the case (b).

Once stated the general conditions for $f_{0}$, let us embed it into a one-parameter family $\left\{f_{\mu}\right\}$ of reversible maps that unfolds generally at $\mu=0$ the initial heteroclinic tangencies at the points of $\Gamma_{12}$. Then, without loss of generality, we can take $\mu$ as the corresponding splitting parameter. By reversibility, the invariant manifolds $W^{u}\left(O_{1}\right)$ and $W^{s}\left(O_{2}\right)$ split as $W^{u}\left(O_{2}\right)$ and $W^{s}\left(O_{1}\right)$ do when $\mu$ varies. Therefore, since these heteroclinic tangencies are quadratic, only one governing parameter is needed to control this splitting.

Let $U$ be an small enough neighbourhood of the contour $C=\left\{O_{1}, O_{2}, \Gamma_{12}, \Gamma_{21}\right\}$. It can be represented as the union of two small neighbourhoods (disks) $U_{1}$ and $U_{2}$ of the saddles $\mathrm{O}_{1}$ and $\mathrm{O}_{2}$ and a finite number of small disks containing those points of $\Gamma_{12}$ and $\Gamma_{21}$ which do not belong to $U_{1}$ and $U_{2}$ (see Figure 2). We will focus our attention on the bifurcations of the so-called single-round periodic orbits, that is, orbits lying entirely in $U$ and having exactly one intersection point with every disk from the set $U \backslash\left(U_{1} \cup U_{2}\right)$. Any point of a single-round periodic orbit is a fixed point of the corresponding first-return map $T_{k m}$, that is constructed by orbits of $f_{\mu}$ with $k$ and $m$ iterations (of $f_{\mu}$ ) in $U_{1}$ and $U_{2}$, respectively. We will call them single-round periodic orbit of type $(k, m)$. However, we do not state the problem of studying the maps $T_{k m}$ for all large $k$ and $m$. We suppose $k$ and $m$ to be large enough integers such that

$$
\lambda_{1}^{k} \simeq \lambda_{2}^{m}
$$

In other words, both values of $\lambda_{1}^{k} \lambda_{2}^{-m}$ and $\lambda_{1}^{-k} \lambda_{2}^{m}$ are uniformly separated from 0 and bounded as $k, m \rightarrow \infty$. The values of $k$ and $m$ are not arbitrary but clearly dependent. The first main result is as follows:

$\ddagger$ However, the property of a symmetric saddle periodic point to be a priori area-preserving is more delicate. It is well-known (see, for instance, [4]) that a symmetric reversible saddle map is "almost conservative" since its (local) analytical normal form and its $C^{\infty}$ formal normal form (up to "flat terms") are conservative.) 
Theorem 1 Let $\left\{f_{\mu}\right\}$ be a one-parameter family of reversible diffeomorphisms that unfolds, generally, at $\mu=0$ the initial heteroclinic tangencies. Assume that $f_{0}$ satisfies conditions $[\mathrm{A}]-[\mathrm{C}]$. Then, in any segment $[-\epsilon, \epsilon]$ with $\epsilon>0$ small, there are infinitely many intervals (not necessarily disjoints)

$$
\delta_{k m}=\left[\mu_{\text {fold }}^{(k, m)}, \mu_{\mathrm{pf}}^{(k, m)}\right] \rightarrow\{0\} \quad \text { as } m, k \rightarrow \infty,
$$

such that the following assertions hold:

(i) The value $\mu=\mu_{\text {fold }}^{(k, m)}$ corresponds to a non-degenerate reversible and selfsymmetric fold bifurcation and, thus, the diffeomorphism $f_{\mu}$ has at $\mu \in \delta_{k m}$ two symmetric, saddle and elliptic, single-round periodic orbits of type $(k, m)$.

(ii) The value $\mu=\mu_{\mathrm{pf}}^{(k, m)}$ corresponds to a symmetric (and non-degenerate if condition [C] holds) pitch-fork bifurcation depending on the type of $f_{0}$ :

$(\text { ii })_{a}$ In the case of "inner" tangency, a symmetric couple of single-round attracting and repelling periodic orbits of type $(k, m)$ is born and, moreover, these orbits undergo simultaneously non-degenerate period doubling bifurcations at the value $\mu=\mu_{\mathrm{pd}}^{(k, m)}$ (where $\mu_{\mathrm{pd}}^{(k, m)} \rightarrow 0$ as $\left.k, m \rightarrow \infty\right)$.

$(i i)_{b}$ For the "outer" tangency, a symmetric couple of contracting-expanding single-round saddle periodic orbits of type $(k, m)$ is born. Moreover, they do not bifurcate any more (at least for $|\mu|<\epsilon$ ).

We refer the reader to Figure 3 for an illustration of this theorem.

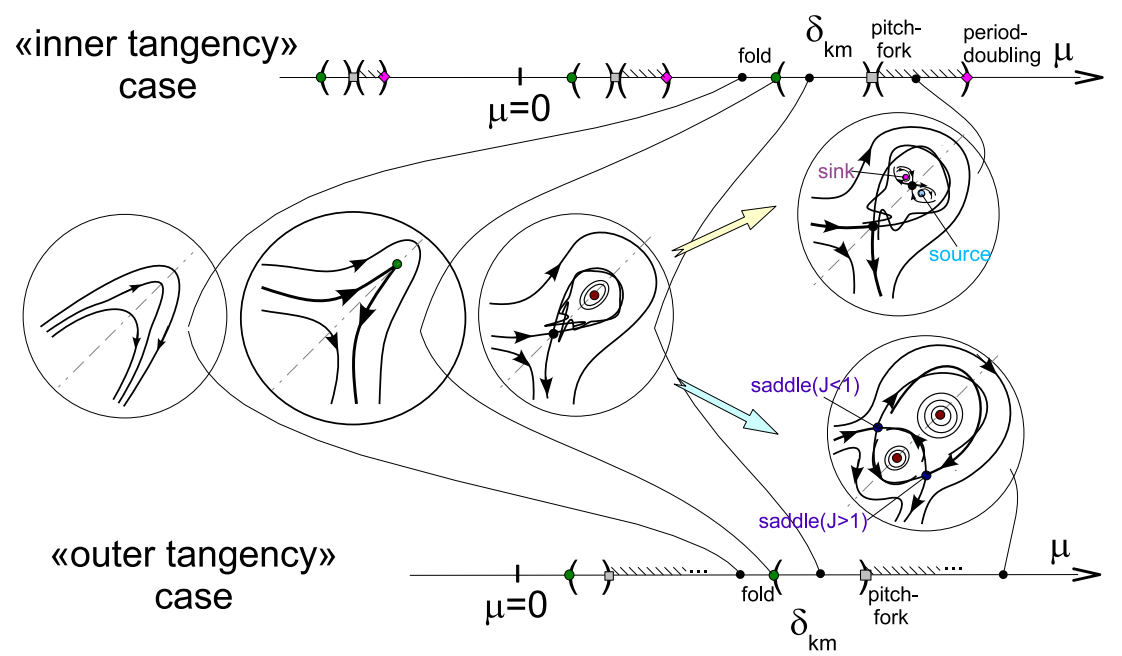

Figure 3. Shadowed intervals correspond to the existence of a symmetric couple of single-round periodic orbits.

Theorem 1 and its counterpart result in [15] show that the appearance of nonconservative periodic orbits under global bifurcations can be considered as a certain general property of two-dimensional reversible maps. 
Briefly, the method we use - based on a rescaling technique - will allow us to prove that the first-return map $T_{k m}$ can be written asymptotically close (as $\left.k, m \rightarrow \infty\right)$ to an area-preserving map of the form:

$$
H:\left\{\begin{array}{l}
\bar{x}=\tilde{M}+\tilde{c} x-y^{2}, \\
\bar{y}=-\frac{\tilde{M}}{\tilde{c}}+\frac{1}{\tilde{c}} y+\frac{1}{\tilde{c}}\left(\tilde{M}+\tilde{c} x-y^{2}\right)^{2},
\end{array}\right.
$$

in which the coordinates $(x, y)$ and the parameters $(\tilde{M}, \tilde{c})$ can take arbitrary values except $\tilde{c}=0$. The region $\tilde{c}<0$ will stand for the "inner" tangency case and $\tilde{c}>0$ for the "outer" one. Its bifurcation diagram is shown in Figure 8. The map (2.3) is, in fact, the product of two Hénon maps with Jacobian $-\tilde{c}$ and $-\tilde{c}^{-1}$. Thus, we can state (see also [31]) that map (2.3) has a complicated dynamics in the corresponding parameter intervals. It can be seen, in particular, that all fixed points become saddles and all of them have homoclinic and heteroclinic intersections for all values of the parameter $\mu$ including (quadratic) tangencies for dense subsets - Newhouse phenomenon. By Lemma 3, analogous "homoclinic tangle" is observed for the first-return map $T_{k m}$ which is reversible again but not conservative in general (see Lemma 4). It means that the map $T_{k m}$ will have four fixed points which are all saddles: two of them are self-symmetric and the other form a couple of symmetric fixed points with Jacobian greater and less than 1. It implies that periodic sinks and sources are involved into chaotic dynamics even in the "outer tangency" case, where the symmetry-breaking pitch-fork bifurcations lead directly to the appearance only of a couple of symmetric saddle periodic points. Thus, we can formulate the following result proving the RMDConjecture for the case under consideration.

Theorem 2 Let $\left\{f_{\mu}\right\}$ be the one-parameter family of reversible maps from Theorem 1. Then, in any segment $[-\varepsilon, \varepsilon]$ of values of $\mu$, there are Newhouse intervals with mixed dynamics connected with an abundance of attracting, repelling and elliptic periodic orbits. This is, values of parameters corresponding to maps $f_{\mu}$ exhibiting simultaneously infinitely many periodic orbits of all these types form a residual set (of second category) in these intervals.

The proof of this Theorem is derived almost immediately from Theorem 1 . Indeed, $\mu$ is the parameter which controls the splitting of the manifolds $W^{u}\left(O_{1}\right)$ and $W^{s}\left(O_{2}\right)$ and, by symmetry, of $W^{u}\left(O_{2}\right)$ and $W^{s}\left(O_{1}\right)$. Thus $\mu=0$ becomes an accumulating point of homoclinic tangencies of $W^{u}\left(O_{1}\right)$ and $W^{s}\left(O_{1}\right)$ and, of course, of $W^{u}\left(O_{2}\right)$ and $W^{s}\left(O_{2}\right)$. From the work of Duarte [5], this implies that $\mu=0$ is an accumulating point for Newhouse regions where the values of the parameter leading to quadratic homoclinic tangencies are dense. By Palis $\lambda$-Lemma, any tangency point between $W^{u}\left(O_{i}\right)$ and $W^{s}\left(O_{i}\right)$ is, in its turn, an accumulating point of pieces of both stable and unstable manifolds. This implies that, in the Newhouse intervals provided by Theorem 1, there exists a dense set of values of $\mu$ giving rise to heteroclinic tangencies between $W^{u}\left(O_{1}\right)$ and $W^{s}\left(O_{2}\right)$ and, by symmetry, between $W^{u}\left(O_{2}\right)$ and $W^{s}\left(O_{1}\right)$. Thus, Theorem 2 follows from the standard procedure of "embedded intervals" associated to the existence of periodic sinks, sources and elliptic points. We refer the reader to $[10,15,13]$ for more details about this procedure.

$\S$ The rescaling method [29] appears to be very efficient to study homoclinic bifurcations. 


\section{Proof of Theorem 1}

\subsection{Preliminary geometric and analytic constructions}

To ease the reading all the proofs of the lemmas of this section have been deferred to Sections 5, 6 and 6.3 .

Let us consider first the map $f_{0}$ and let $M_{1}^{-} \in U_{1}, M_{2}^{+} \in U_{2}$ be a pair of points of the orbit $\Gamma_{12}$ and $M_{2}^{-} \in U_{2}, M_{1}^{+} \in U_{1}$ be a pair of points of $\Gamma_{21}$. Consider $\Pi_{i}^{+} \subset U_{i}$ and $\Pi_{i}^{-} \subset U_{i}$ small neighbourhoods of the heteroclinic points $M_{i}^{+}$and $M_{i}^{-}$(see Figure 4 ). One can always take $U_{i}, \Pi_{i}^{-}, \Pi_{i}^{+}, i=1,2$ to be also $R$-symmetric (that is $\left.R\left(U_{i}\right)=U_{i}, R\left(\Pi_{i}^{-}\right)=\Pi_{i}^{-}\right)$. Let us assume that $(i)$ the heteroclinic points are symmetric under the involution $R$, i.e. $M_{1}^{-}=R\left(M_{1}^{+}\right)$and $M_{2}^{-}=R\left(M_{2}^{+}\right)$, and $(i i)$ they are the "last" points on $U_{1}$ and $U_{2}$, that is, $f_{0}\left(M_{i}^{-}\right) \notin U_{i}$ (and, thus, $\left.f_{0}^{-1}\left(M_{i}^{+}\right) \notin U_{i}\right)$. Let $q$ be such an positive integer that $M_{2}^{+}=f_{0}^{q}\left(M_{1}^{-}\right)$(and, thus, $\left.M_{1}^{+}=f_{0}^{q}\left(M_{2}^{-}\right)\right)$.

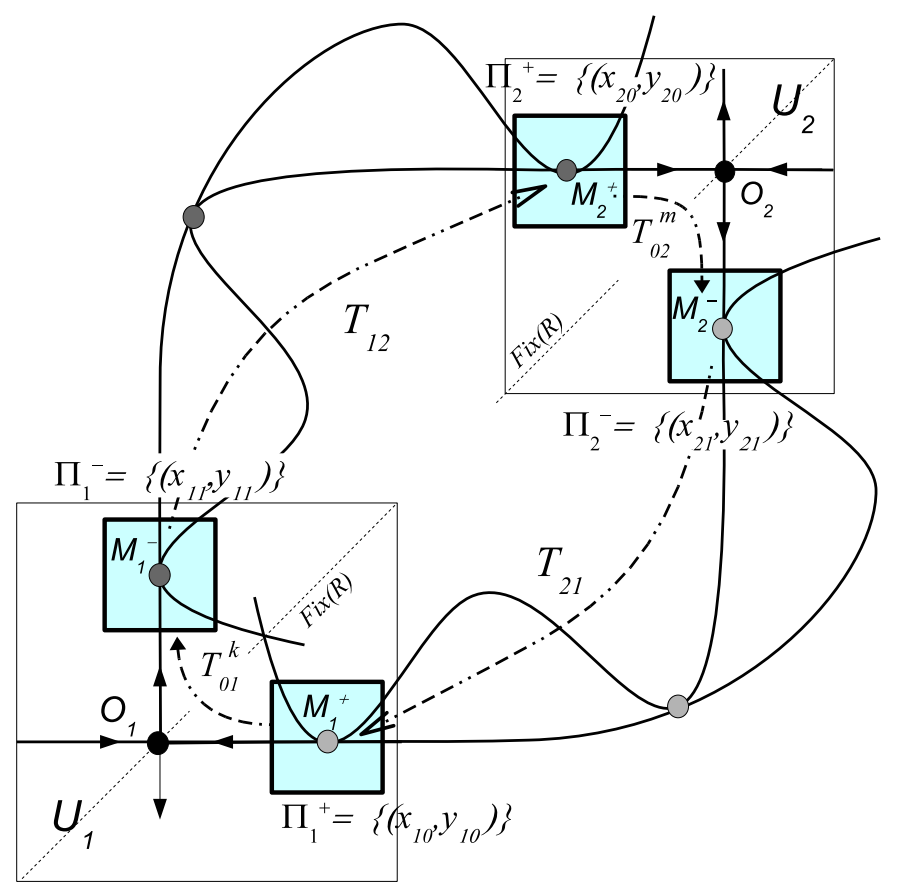

Figure 4. Schematic actions of the local $\left(T_{01}^{k}\right.$ and $\left.T_{02}^{m}\right)$ and global $\left(T_{12}\right.$ and $\left.T_{21}\right)$ maps in the neighbourhood $U$ of the contour $C=\left\{O_{1}, O_{2}, \Gamma_{12}, \Gamma_{21}\right\}$.

Consider now the map $f_{\mu}$. Denote $\left.T_{0 i} \equiv f_{\mu}\right|_{U_{i}}, i=1,2$. The $\mu$-dependent maps $T_{01}$ and $T_{02}$ are called the local maps. We introduce also the so-called global maps $T_{12}$ and $T_{21}$ by the following relations: $T_{12} \equiv f_{\mu}^{q}: \Pi_{1}^{-} \rightarrow \Pi_{2}^{+}$and $T_{12} \equiv f_{\mu}^{q}: \Pi_{2}^{-} \rightarrow \Pi_{1}^{+}$ (see Figure 4). Then the first-return map $T_{k m}: \Pi_{1}^{+} \mapsto \Pi_{1}^{+}$is defined by the following composition of maps and neighbourhoods:

$$
\Pi_{1}^{+} \stackrel{T_{01}^{k}}{\longrightarrow} \Pi_{1}^{-} \stackrel{T_{12}}{\longrightarrow} \Pi_{2}^{+} \stackrel{T_{02}^{m}}{\longrightarrow} \Pi_{2}^{-} \stackrel{T_{21}}{\longrightarrow} \Pi_{1}^{+}
$$


Denote local coordinates on $\Pi_{i}^{+}$and $\Pi_{i}^{-}$as $\left(x_{0 i}, y_{0 i}\right)$ and $\left(x_{1 i}, y_{1 i}\right)$, respectively. Then the chain (3.1) can be represented as

$$
\left(x_{01}, y_{01}\right) \stackrel{T_{01}^{k}}{\longmapsto}\left(x_{11}, y_{11}\right) \stackrel{T_{12}}{\longmapsto}\left(x_{02}, y_{02}\right) \stackrel{T_{02}^{m}}{\longmapsto}\left(x_{12}, y_{12}\right) \stackrel{T_{21}}{\longmapsto}\left(\bar{x}_{01}, \bar{y}_{01}\right) .
$$

As usual, we need such local coordinates on $U_{1}$ and $U_{2}$ in which the maps $T_{01}$ and $T_{02}$ have their simplest form. We can not assume the maps $T_{0 i}$ are linear, since by condition [A], only $C^{1}$-linearisation is possible here. Therefore, we consider such $C^{r-1}$-coordinates in which the local maps have the so-called main normal form or first order normal form.

Lemma 1 (Main normal form of a saddle map) Let a $C^{r}$-smooth map $T_{0}$ be reversible with $\operatorname{dim} \operatorname{Fix} T_{0}=1$. Suppose that $T_{0}$ has a saddle fixed (periodic) point $O$ belonging to the line Fix $T_{0}$ and having multipliers $\lambda$ and $\lambda^{-1}$, with $|\lambda|<1$. Then there exist $C^{r-1}$-smooth local coordinates near $O$ in which the map $T_{0}$ (or $T_{0}^{n}$, where $n$ is the period of $O$ ) can be written in the following form:

$$
\begin{array}{ll}
T_{0}: & \bar{x}=\lambda x\left(1+h_{1}(x, y) x y\right) \\
& \bar{y}=\lambda^{-1} y\left(1+h_{2}(x, y) x y\right),
\end{array}
$$

where $h_{1}(0,0)=-h_{2}(0,0)$. The map (3.2) is reversible with respect to the standard linear involution $(x, y) \mapsto(y, x)$. In fact, it can be expressed in the so-called cross-form (see Section 5.1):

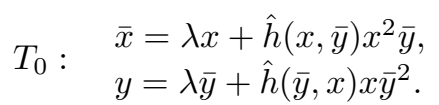

The proof of this lemma has been deferred to Subsection 5.2.

In the case that $T_{0}$ is linear, i.e. of the form $\bar{x}=\lambda x, \bar{y}=\lambda^{-1} y$, its $j$-th iterates $\left(x_{j}, y_{j}\right)=T_{0}^{j}\left(x_{0}, y_{0}\right)$ are given simply by $x_{j}=\lambda^{j} x_{0}, y_{j}=\lambda^{-j} y_{0}$ in standard explicit form or by $x_{j}=\lambda^{j} x_{0}, y_{0}=\lambda^{j} y_{j}$ in cross-form. If $T_{0}$ is nonlinear such cross-form expression for $T_{0}^{j}$ exists too. Precisely, the following result holds:

Lemma 2 (Iterations of the local map) Let $T_{0}$ be a saddle map written in the main normal form (3.2) (or (3.3)) in a small neighbourhood $V$ of $O$. Let us consider points $\left(x_{0}, y_{0}\right), \ldots,\left(x_{j}, y_{j}\right)$ from $V$ such that $\left(x_{l+1}, y_{l+1}\right)=T_{0}\left(x_{l}, y_{l}\right), l=0, \ldots, j-1$. Then one has

$$
\begin{aligned}
& x_{j}=\lambda^{j} x_{0}\left(1+j \lambda^{j} h_{j}\left(x_{0}, y_{j}\right)\right), \\
& y_{0}=\lambda^{j} y_{j}\left(1+j \lambda^{j} h_{j}\left(y_{j}, x_{0}\right)\right),
\end{aligned}
$$

where the functions $h_{j}\left(y_{j}, x_{0}\right)$ are uniformly bounded with respect to $j$ as well as all their derivatives up to order $r-2$.

Remark 1 (a) Both lemmas 1 and 2 are true if $T_{0}$ depends on parameters. Moreover, if the initial $T_{0}$ is $C^{r}$ with respect to coordinates and parameters, then the normal form (3.2) is $C^{r-1}$ with respect to coordinates and $C^{r-2}$ with respect to parameters (see [14], Lemmas 6 and 7).

(b) Bochner Theorem (see [16]) ensures that any involution $R$ with $\operatorname{dim} \operatorname{Fix} R=1$ is locally smoothly conjugated to its linear part around a symmetric point. It is not a loss of generality to assume that maps $f_{\mu}$ are reversible under an involution $R$ with linear part given by $L(x, y)=(y, x)$. As it will be shown, this fact will be very convenient in the construction of the local maps $T_{01}$ and $T_{02}$. 
(c) Similar results related to finite-smooth normal forms of saddle maps were established in [7, 11, 12, 13] for general, near-conservative and conservative maps. The proof of our lemmas are just an adapted version to the reversible setting of their proofs.

\subsection{Construction of the local and global maps}

By Lemma 1 one can choose in $U_{1}$ and $U_{2}$ local coordinates $\left(x_{1}, y_{1}\right)$ and $\left(x_{2}, y_{2}\right)$, respectively, such that the maps $T_{01}$ and $T_{02}$ take the following form:

$$
T_{01}: \quad \bar{x}_{1}=\lambda_{1} x_{1}+h_{1}^{1}\left(x_{1}, y_{1}\right) x_{1}^{2} y_{1}, \quad \bar{y}_{1}=\lambda_{1}^{-1} y_{1}+h_{2}^{1}\left(x_{1}, y_{1}\right) x_{1} y_{1}^{2},
$$

and

$$
T_{02}: \quad \bar{x}_{2}=\lambda_{2} x_{2}+h_{1}^{2}\left(x_{2}, y_{2}\right) x_{2}^{2} y_{2}, \quad \bar{y}_{2}=\lambda_{2}^{-1} y_{2}+h_{2}^{2}\left(x_{2}, y_{2}\right) x_{2} y_{2}^{2} .
$$

Furthermore, in these coordinates, the local stable and unstable invariant manifolds of both points $O_{1}$ and $O_{2}$ are straightened: $x_{i}=0$ is the equation of $W_{l o c}^{u}\left(O_{i}\right)$ and $y_{i}=0$ is the equation of $W_{l o c}^{s}\left(O_{i}\right), i=1,2$. Then, we can write the $(x, y)$-coordinates of the chosen heteroclinic points as follows: $M_{1}^{+}=\left(x_{1}^{+}, 0\right), M_{1}^{-}=\left(0, y_{1}^{-}\right), M_{2}^{+}=\left(x_{2}^{+}, 0\right)$ and $M_{2}^{-}=\left(0, y_{2}^{-}\right)$. Besides, because of the reversibility, we have that

$$
x_{1}^{+}=y_{1}^{-}=\alpha_{1}^{*}, \quad x_{2}^{+}=y_{2}^{-}=\alpha_{2}^{*}
$$

We assume that $T_{0 i}\left(\Pi_{i}^{+}\right) \cap \Pi_{i}^{+}=\emptyset$ and $T_{0 i}^{-1}\left(\Pi_{i}^{-}\right) \cap \Pi_{i}^{-}=\emptyset, i=1,2$. Then the domain of definition of the successor map from $\Pi_{i}^{+}$into $\Pi_{i}^{-}$under iterations of $T_{0 i}$ consists of infinitely many non-intersecting strips $\sigma_{j}^{0 i}$ which belong to $\Pi_{i}^{+}$and accumulate at $W_{l o c}^{s}\left(O_{i}\right) \cap \Pi_{i}^{+}$as $j \rightarrow \infty$. Analogously, the range of this map consists of infinitely many strips $\sigma_{j}^{1 i}=T_{0 i}^{j}\left(\sigma_{j}^{0 i}\right)$ belonging to $\Pi_{i}^{-}$and accumulating at $W_{l o c}^{u}\left(O_{i}\right) \cap \Pi_{i}^{-}$as $j \rightarrow \infty$ (see Figure 5).

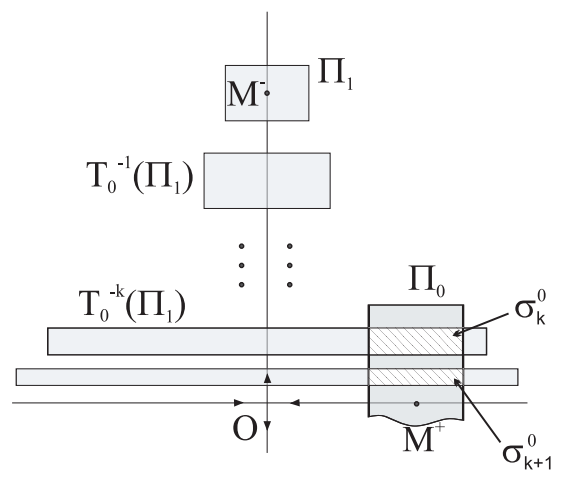

(a)

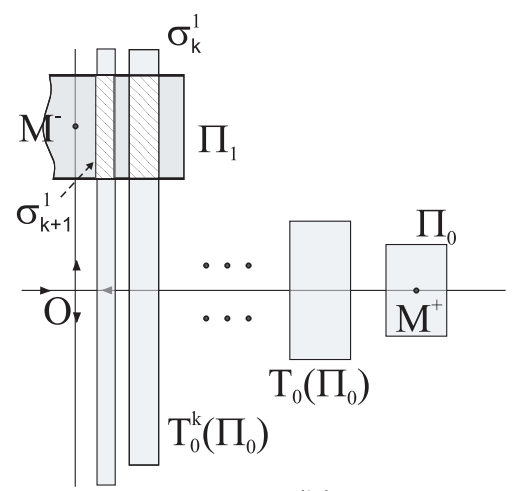

(b)

Figure 5. A geometry of creation of both domains of definition $\sigma_{i}^{0} \subset \Pi^{+}$(a) and domains of the range $\sigma_{i}^{1} \subset \Pi^{-}(\mathrm{b})$ for the maps $T_{0}^{i}: \Pi^{+} \rightarrow \Pi^{-}$.

It follows from Lemma 2 that the map $T_{01}^{k}: \sigma_{k}^{01} \mapsto \sigma_{k}^{11}$ can be written in the following form (for large enough values of $k$ )

$$
T_{01}^{k}:\left\{\begin{array}{l}
x_{11}=\lambda_{1}^{k} x_{01}\left(1+k \lambda_{1}^{k} h_{k}^{1}\left(x_{01}, y_{11}\right)\right) \\
y_{01}=\lambda_{1}^{k} y_{11}\left(1+k \lambda_{1}^{k} h_{k}^{1}\left(y_{11}, x_{01}\right)\right)
\end{array}\right.
$$


and an analogous formula takes place for the map $T_{02}^{m}: \sigma_{m}^{02} \mapsto \sigma_{m}^{12}$ :

$$
T_{02}^{m}:\left\{\begin{array}{l}
x_{12}=\lambda_{2}^{m} x_{02}\left(1+m \lambda_{2}^{m} h_{m}^{2}\left(x_{02}, y_{12}\right)\right) \\
y_{02}=\lambda_{2}^{m} y_{12}\left(1+m \lambda_{2}^{m} h_{m}^{2}\left(y_{12}, x_{02}\right)\right)
\end{array}\right.
$$

We write now the global map $T_{12}$ in the following form

$$
T_{12}\left\{\begin{array}{c}
x_{02}-x_{2}^{+}=F_{12}\left(x_{11}, y_{11}-y_{1}^{-}, \mu\right) \equiv a x_{11}+b\left(y_{11}-y_{1}^{-}\right)+ \\
l_{02}\left(y_{11}-y_{1}^{-}\right)^{2}+\varphi_{1}\left(x_{11}, y_{11}, \mu\right) \\
y_{02}=G_{12}\left(x_{11}, y_{11}-y_{1}^{-}, \mu\right) \equiv \mu+c x_{11}+d\left(y_{11}-y_{1}^{-}\right)^{2}+ \\
f_{11} x_{11}\left(y_{11}-y_{1}^{-}\right)+f_{03}\left(y_{11}-y_{1}^{-}\right)^{3}+ \\
\varphi_{2}\left(x_{11}, y_{11}, \mu\right)
\end{array}\right.
$$

where $F_{12}(0)=G_{12}(0)=0$ since $T_{12}\left(M_{1}^{-}\right)=M_{2}^{+}$at $\mu=0$ and

$$
\begin{aligned}
& \varphi_{1}=O\left(\left|y_{11}-y_{1}^{-}\right|^{3}\right)+x_{11} O\left(\left\|\left(x_{11}, y_{11}-y_{1}^{-}\right)\right\|\right), \\
& \varphi_{2}=O\left(\left|x_{11}\right|^{2}\right)+O\left(\left|y_{11}-y_{1}^{-}\right|^{4}\right)+O\left(x_{11}\left(y_{11}-y_{1}^{-}\right)^{2}\right) .
\end{aligned}
$$

Since the curves $T_{12}\left(W_{l o c}^{u}\left(O_{1}\right):\left\{x_{11}=0\right\}\right)$ and $W_{l o c}^{s}\left(O_{2}\right):\left\{y_{02}=0\right\}$ have a quadratic tangency at $\mu=0$, it implies that

$$
\frac{\partial G_{12}(0)}{\partial y_{11}}=0, \quad \frac{\partial^{2} G_{12}(0)}{\partial y_{11}^{2}}=2 d \neq 0 .
$$

The Jacobian $J\left(T_{12}\right)$ has, obviously, the following form:

$$
J\left(T_{12}\right)=-b c+a f_{11} x_{11}+Q\left(y_{11}-y_{1}^{-}\right)+O\left(x_{11}^{2}+\left(y_{11}-y_{1}^{-}\right)^{2}\right),
$$

where

$$
Q=2 a d-b f_{11}-2 c l_{02} .
$$

Now condition [C] can be formulated more precisely. Namely, we require that

$$
Q=\left.\frac{\partial J\left(T_{12}\right)}{\partial y_{11}}\right|_{\left(x_{11}=0, y_{11}=y_{1}^{-}, \mu=0\right)} \neq 0
$$

Concerning the global map $T_{21}$, we cannot write it now in an arbitrary form. The point is that after written a formula for the map $T_{12}$ it is necessary to use the reversibility relations to get the one associated to it:

$$
T_{21}=R T_{12}^{-1} R^{-1}, \quad T_{12}=R T_{21}^{-1} R^{-1}
$$

for constructing $T_{21}$. Then, by (3.7), we obtain that the map $T_{21}^{-1}: \Pi_{1}^{+}\left\{\left(x_{01}, y_{01}\right)\right\} \mapsto$ $\Pi_{2}^{-}\left\{\left(x_{12}, y_{12}\right)\right\}$ must be written as follows

$$
T_{21}^{-1}\left\{\begin{array}{c}
x_{12}=G_{12}\left(y_{01}, x_{01}-y_{1}^{-}, \mu\right)= \\
\mu+c y_{01}+d\left(x_{01}-y_{1}^{-}\right)^{2}+f_{11} y_{01}\left(x_{01}-y_{1}^{-}\right)+ \\
f_{13}\left(x_{01}-y_{1}^{-}\right)^{2}+\varphi_{2}\left(y_{01}, x_{01}, \mu\right) \\
y_{12}-x_{2}^{+}=F_{12}\left(y_{01}, x_{01}-y_{1}^{-}, \mu\right)= \\
a y_{01}+b\left(x_{01}-y_{1}^{-}\right)+l_{02}\left(x_{01}-y_{1}^{-}\right)^{2}+\varphi_{1}\left(y_{01}, x_{01}, \mu\right)
\end{array}\right.
$$

Relation (3.9) allows to define the map $T_{21}: \Pi_{2}^{-}\left\{\left(x_{12}, y_{12}\right)\right\} \mapsto \Pi_{1}^{+}\left\{\left(x_{01}, y_{01}\right)\right\}$, but in implicit form. 


\subsection{Construction of the first-return maps $T_{k m}$ and the Rescaling Lemma}

Using relations (3.6)-(3.9), we can construct the first-return map $T_{k m}=T_{21} T_{02}^{m} T_{12} T_{01}^{k}$ defined on the strip $\sigma_{k}^{01} \subset \Pi_{1}^{+}$. Recall that any fixed point of $T_{k m}$ corresponds to a single-round periodic orbit of type $(k, m)$ of period $(k+m+2 q)$. We suppose $k$ and $m$ to be large enough integers satisfying the relation (2.2). Then the following result holds.

Lemma 3 (The rescaling lemma) Let the map $f_{0}$ satisfy conditions [A]-[B] and $\left\{f_{\mu}\right\}$ be a general unfolding in the class of reversible maps. Suppose $k$ and $m$ are large enough integer numbers satisfying relation (2.2). Then there exist coordinates (called "rescaled coordinates") in such a way that the first-return map $T_{k m}:(x, y) \mapsto(\bar{x}, \bar{y})$ is given implicitly by

$$
\begin{aligned}
& M+c \bar{y}+d \bar{x}^{2}+f_{11} \lambda_{1}^{k} \bar{x} \bar{y}+f_{03} \lambda_{1}^{k} \bar{x}^{3}= \\
& \quad=b \lambda_{2}^{m} \lambda_{1}^{-k} y+a \lambda_{2}^{m} x+l_{02} \lambda_{2}^{m} y^{2}+O\left(k \lambda_{1}^{2 k}\right), \\
& M+c x+d y^{2}+f_{11} \lambda_{1}^{k} x y+f_{03} \lambda_{1}^{k} y^{3}= \\
& b \lambda_{2}^{m} \lambda_{1}^{-k} \bar{x}+a \lambda_{2}^{m} \bar{y}+l_{02} \lambda_{2}^{m} \bar{x}^{2}+O\left(k \lambda_{1}^{2 k}\right),
\end{aligned}
$$

where

$$
M=\lambda_{1}^{-2 k}\left(\mu+c \lambda_{1}^{k} \alpha_{1}^{*}(1+\ldots)-\lambda_{2}^{m} \alpha_{2}^{*}(1+\ldots)\right)
$$

and "..." stands for some coefficients tending to zero as $k, m \rightarrow \infty$. Moreover, the domain of definition of the new coordinates $x$ and $y$ and parameter $M$ cover all finite values as $k, m \rightarrow \infty$.

\subsection{On bifurcations of fixed points of the first-return maps $T_{k m}$}

We will approach the study of bifurcations of the first-return map $T_{k m}$ through its rescaled form (3.10). Indeed, neglecting in the latter equation all the asymptotically small terms (as $k, m \rightarrow \infty$ ), we obtain the following truncated form for $T_{k m}$

$$
\left\{\begin{aligned}
M+c \bar{y}+d \bar{x}^{2} & =\beta_{k m} y \\
\beta_{k m} \bar{x} & =M+c x+d y^{2}
\end{aligned}\right.
$$

given in cross-form and where $\beta_{k m}=b \lambda_{1}^{-k} \lambda_{2}^{m} \neq 0$ is, by hypothesis (see (2.2)), a coefficient which is uniformly bounded from zero and infinity for all admissible $k$ and $m$. Introducing the following rescaled coordinates

$$
x=-\frac{\beta_{k m}}{d} x_{\text {new }}, \quad y=-\frac{\beta_{k m}}{d} y_{\text {new }} .
$$

and denoting again $\left(x_{\text {new }}, y_{\text {new }}\right)$ as $(x, y)$, we reach the following map (in cross-form)

$$
H:\left\{\begin{aligned}
\tilde{M}+\tilde{c} \bar{y}-\bar{x}^{2} & =y, \\
\bar{x} & =\tilde{M}+\tilde{c} x-y^{2},
\end{aligned}\right.
$$

with

$$
\tilde{M}=-\frac{d}{\beta_{k m}^{2}} M, \quad \tilde{c}=\frac{c}{\beta_{k m}}=\frac{c}{b} \lambda_{1}^{k} \lambda_{2}^{-m}
$$

Condition $c \neq 0$ implies two different scenarios for $H$ in space of parameters $(\tilde{c}, \tilde{M})$, depending on the sign of $\tilde{c}$. 
Observe that the map $H$ can be expressed in the explicit form (2.3) and, moreover, it can be represented as the superposition $H=\mathcal{H}_{2} \circ \mathcal{H}_{1}$ of two Hénon maps

$$
\mathcal{H}_{1}:\left\{\begin{array}{l}
\bar{x}=y \\
\bar{y}=\tilde{M}+\tilde{c} x-y^{2}
\end{array}\right.
$$

and

$$
\mathcal{H}_{2}:\left\{\begin{aligned}
\bar{x} & =y \\
\bar{y} & =-\frac{\tilde{M}}{\tilde{c}}+\frac{1}{\tilde{c}} x+\frac{1}{\tilde{c}} y^{2}
\end{aligned}\right.
$$

The Jacobians of these maps are constant and inverse one of each other: $J\left(\mathcal{H}_{1}\right)=-\tilde{c}$ and $J\left(\mathcal{H}_{2}\right)=-\tilde{c}^{-1}$. Therefore, the resulting map $H$ has Jacobian equal to 1 and is, therefore, area-preserving.

The symmetric form (3.13) of $H$ provides a simple geometric interpretation for its fixed points. Indeed, they must satisfy

$$
y(1-\tilde{c})=\tilde{M}-x^{2}, \quad x(1-\tilde{c})=\tilde{M}-y^{2},
$$

which correspond, for $\tilde{c} \neq 1$, to the intersection points of a symmetric (with respect to the bisectrix $x=y$ ) couple of parabolas. For any fixed value of $\tilde{c} \neq 1$ varying $\tilde{M}$ one obtain several possible intersections. For instance, in the case $\tilde{c}<1$ (represented

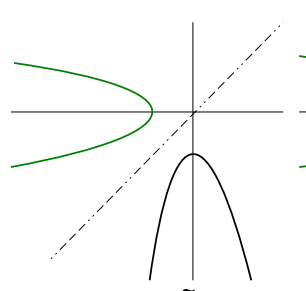

(a) $\tilde{M}<M_{1}{ }^{*}$

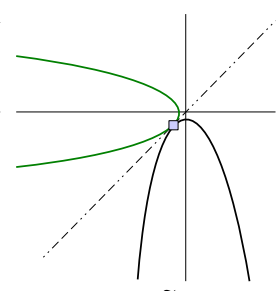

(b) $\tilde{M}=M_{1}^{*}$

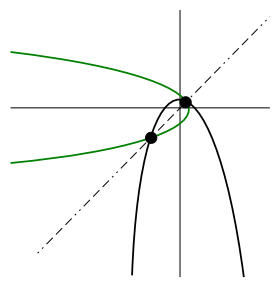

(c) $\mathrm{M}_{1}{ }^{*}<\tilde{\mathrm{M}}<\mathrm{M}_{1}{ }^{* *}$

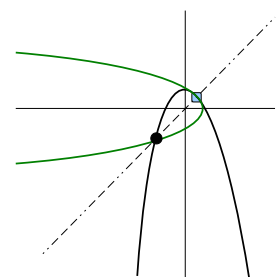

(d) $\tilde{M}=M_{1}^{* *}$

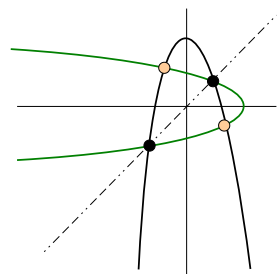

(e) $\tilde{M}>M_{1}^{* *}$

Figure 6. A geometric interpretation of the fixed points of map $H$ in (3.13) as intersection points of a symmetric couple of parabolas (case $\tilde{c}<1$ )

in Figures 6 and 7), the following five situations are given:

(a) the two parabolas do not intersect if $\tilde{M}<M_{1}^{*}=-\frac{1}{4}(\tilde{c}-1)^{2}$;

(b) they have a quadratic contact among them and with the bisectrix when $\tilde{M}=M_{1}^{*}$;

(c) they have two symmetric (that is, belonging to its symmetry line $x=y$ ) intersection points provided $M_{1}^{*}<\tilde{M}<M_{2}^{*}=\frac{3}{4}(\tilde{c}-1)^{2}$;

(d) they present a cubic symmetric tangency when $\tilde{M}=M_{2}^{*}$ and, finally, 
(e) the two parabolas intersect in four points if $\tilde{M}>M_{2}^{*}$ : two symmetric points and a symmetric couple of points.

A similar picture takes place for $\tilde{c}>1$. However, case $\tilde{c}=1$ is very special: equation (3.15) becomes $0=\tilde{M}-x^{2}, 0=\tilde{M}-y^{2}$ and, therefore a kind of " $0-4$ "bifurcation occurs at $\tilde{M}=0$. There are no fixed points of $H$ for $\tilde{M}<0$ and four fixed points appear for $\tilde{M}>0$.

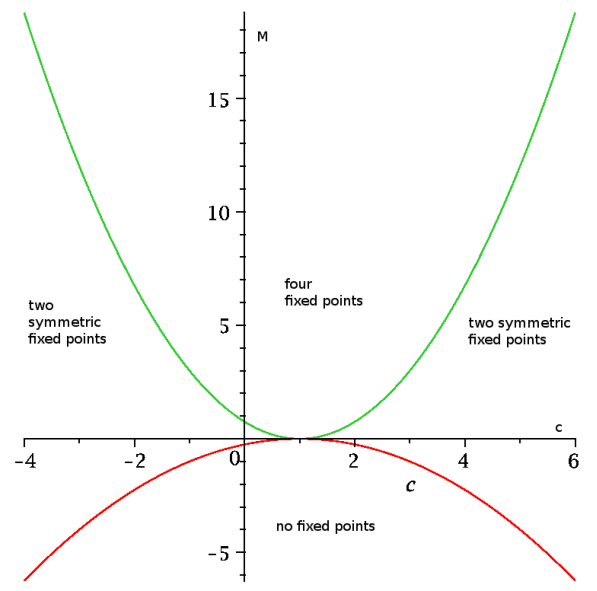

Figure 7. Regions of fixed points of map $H$ in the space of parameters $(\tilde{c}, \tilde{M})$

Let us show with same detail the bifurcations one finds when varying the parameters $(\tilde{c}, \tilde{M})$. We divide this study in two cases: $\tilde{c}<0$ and $\tilde{c}>0$ (which contains, in its turn, the cases $0<\tilde{c}<1, \tilde{c}=1$ and $\tilde{c}>1$ ). We start with the first one.

- Case $\tilde{c}<0$ :

As we have shown above, no fixed points of map $H$ appear for a fixed $\tilde{c}<0$ until the parameter $\tilde{M}$ reaches the value $\tilde{M}^{*}$. At that point, a fold bifurcation occurs. This bifurcation is characterized by the appearance of two fixed points, saddle and elliptic ones, and has the equation

$$
F_{1,2}: \quad \tilde{M}=-\frac{1}{4}(\tilde{c}-1)^{2}
$$

(this curve is denoted in Figure 8 by $F_{1}$ and $F_{2}$, associated to $\tilde{c}<0$ and $\tilde{c}>0$, respectively). If we call $P_{+}=\left(p_{+}, p_{+}\right)$and $P_{-}=\left(p_{-}, p_{-}\right)$the (symmetric) fixed points, it is straightforward to check that they are given by

$$
p_{ \pm}=\frac{\tilde{c}-1 \pm \sqrt{(\tilde{c}-1)^{2}+4 \tilde{M}_{1}^{*}}}{2},
$$

where we recall that $\tilde{M}_{1}^{*}=-\frac{1}{4}(\tilde{c}-1)^{2}$.

The point $P_{-}$will remain a saddle for any value of $\tilde{M} \geq \tilde{M}^{*}$ while point $P_{+}$will undergo several bifurcations. Precisely, period-doubling bifurcations occur when 
the parameter $\tilde{M}$ crosses the curves

$$
\begin{aligned}
& P D^{1}\left(P_{+}\right): \quad \tilde{M}=1-\frac{1}{4}(\tilde{c}-1)^{2}, \\
& P D^{3}\left(P_{+}\right): \quad \tilde{M}=\frac{(\tilde{c}+1)(3 \tilde{c}-1)}{4} .
\end{aligned}
$$

In Figure 8 some "grey arrows" indicate the directions of birth of these period-2 points.

Besides these period-doubling bifurcations, a pitch-fork bifurcation curve exists for this point $P_{+}$. Indeed, when crossing the curve

$$
P F^{1}\left(P_{+}\right): \quad \tilde{M}=\frac{3}{4}(\tilde{c}-1)^{2},
$$

(from region $\mathrm{V}_{l}$ to region $\mathrm{VI}_{l}$ in Figure 8) the point $P_{+}$becomes a saddle fixed point - that we denote again by $P_{+}$- and a symmetric couple of elliptic fixed points $P_{3}$ and $P_{4}$ appear. When increasing $\tilde{M}$, the point $P_{+}$does not bifurcate any more whereas the points $P_{3}$ and $P_{4}$ undergo simultaneously period-doubling bifurcation at crossing the curve

$$
P D\left(P_{3,4}\right): \quad \tilde{M}=\frac{(1-3 \tilde{c})(3-\tilde{c})}{4} .
$$

Further variation of parameters at the region $\mathrm{VII}_{l}$ in Figure 8 will lead to a cascade of period-doubling bifurcations of symmetric couples of periodic points.

- Case $\tilde{c}>0$ :

As it has been mentioned for the case $\tilde{c}<0$, a fold-bifurcation curve is found at the curve

$$
\tilde{M}=-\frac{1}{4}(\tilde{c}-1)^{2},
$$

denoted for $\tilde{c}>0$ by $F_{2}$ in Figure 8 . In this case, both $P_{-}=\left(p_{-}, p_{-}\right)$and $P_{+}=\left(p_{+}, p_{+}\right)$fixed points, given by (3.17), undergo pitch-fork and perioddoubling bifurcations. Namely, for $P_{+}$, these bifurcations curves are, respectively,

$$
\begin{aligned}
& P F^{2}\left(P_{+}\right): \quad \tilde{M}=\frac{3}{4}(\tilde{c}-1)^{2} \quad \text { for } 0<\tilde{c}<1, \\
& P D^{2}\left(P_{+}\right): \quad \tilde{M}=1-\frac{1}{4}(\tilde{c}-1)^{2}, \quad \text { for } \tilde{c}>0 .
\end{aligned}
$$

Regarding the point $P_{-}$one has the following pitch-fork and period-doubling bifurcation curves:

$$
\begin{aligned}
& P F\left(P_{-}\right): \quad \tilde{M}=\frac{3}{4}(\tilde{c}-1)^{2} \quad \text { for } \tilde{c}>1, \\
& P D\left(P_{-}\right): \quad \tilde{M}=\frac{(\tilde{c}+1)(3 \tilde{c}-1)}{4} \quad \text { for } \tilde{c}>0 .
\end{aligned}
$$

When crossing the (pitch-fork bifurcation) curve $P F^{2}\left(P_{+}\right) \cup P F\left(P_{-}\right)$, a symmetric couple of saddle fixed points $P_{3}$ and $P_{4}$ are born but they do not bifurcate any more when increasing the value of $\tilde{M}$.

One can expect, however, that bifurcations of the symmetric (elliptic) fixed points $P_{+}$and $P_{-}$give rise to cascades of period-doubling bifurcations [32]. 


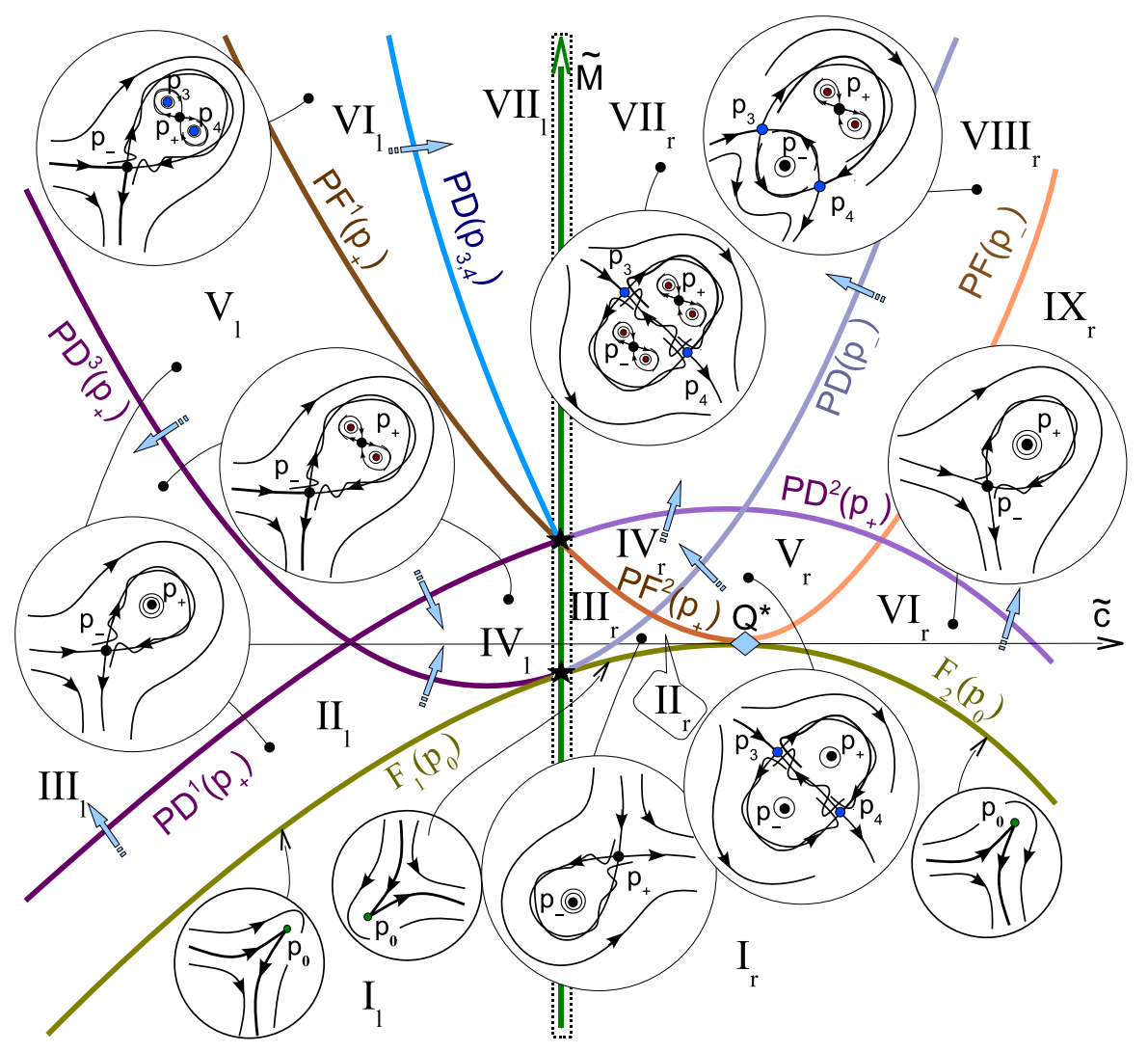

Figure 8. Elements of the bifurcation diagram for the map $H$.

Even though map $H$ is a truncation of the map $T_{k m}$, it is important to stress that its behavior around the fixed points appearing from the pitch-fork, perioddoubling and fold bifurcations is not exactly the same. Indeed, pitch-fork and perioddoubling bifurcations can lead in $T_{k m}$ to a slightly different behavior of the truncated area-preserving map $H$. Namely, pitch-fork bifurcations in $T_{k m}$ lead again to the appearance of two non-symmetric fixed points $P_{3}$ and $P_{4}$ but these points can be non-conservative. In fact, this seems to be a quite general situation as the following lemma shows.

Lemma 4 (Non-conservative fixed points) The non-symmetric fixed points $P_{3}$ and $P_{4}$ of map (3.10), with $k$ and $m$ satisfying (2.2), have Jacobian $J_{n s}$ and $J_{n s}^{-1}$, respectively, with

$$
J_{n s}=1+\frac{Q\left(\eta^{*}-\xi^{*}\right)}{b c} \lambda_{1}^{k}+o\left(\lambda_{1}^{k}\right)
$$

where $Q$ is the coefficient given by formula (3.8) and $\xi^{*}$ and $\eta^{*}$ are, respectively, the $x$ - and $y$-coordinate of the fixed point $\left(P_{3}\right.$ and $\left.P_{4}\right)$.

On the other hand, fold bifurcations of the map $T_{k m}$ lead, similarly to what happens for the map $H$, to the appearance of two symmetric (saddle and elliptic) fixed points 
$P_{+}$and $P_{-}$. Concerning the symmetric elliptic fixed point, we have the following result.

Lemma 5 (Symmetric elliptic fixed points) The point $P_{+}$is generic elliptic (i.e. $K A M$-stable) for open and dense sets of values of the parameters $(\tilde{c}, \tilde{M})$ in the domains $I I_{l} \cup V_{l}$ and $I V_{r} \cup V_{r} \cup V I_{r}$. The same happens for the elliptic point $P_{-}$in the domain $I I_{r} \cup V_{r} \cup V I I I_{r}$.

The proofs of lemmas 3 to 5 have been deferred to Section 6.3.

\subsection{End of the proof of Theorem 1.}

If $k$ and $m$ are large enough integers satisfying (2.2) and having in mind expressions (3.11) and (3.14), the following relation between the parameters $\mu$ and $\tilde{M}$ holds:

$$
\mu=\lambda_{2}^{m} \alpha_{2}^{*}\left(1+\rho_{k}^{1}\right)-c \lambda_{1}^{k} \alpha_{1}^{*}\left(1+\rho_{k}^{2}\right)-\frac{b^{2}}{d} \tilde{M} \lambda_{2}^{2 m}\left(1+\rho_{k}^{3}\right),
$$

where $\rho_{k}^{i}, i=1,2,3$, are some small coefficients $\left(\rho_{k}^{i} \rightarrow 0\right.$ as $\left.k \rightarrow \infty\right)$. Using formulas (3.16)-(3.18) for the bifurcation curves of the truncated map (3.13), asymptotically close to the (rescaled) first-return map (3.10), we find the following expressions for the bifurcation values of $\mu$ at the statement of Theorem 1: a value $\mu=\mu_{\text {fold }}^{(k, m)}$, which corresponds to the fold bifurcation in $T_{k m}$,

$$
\begin{aligned}
\mu_{\mathrm{fold}}^{(k, m)}= & \lambda_{2}^{m} \alpha_{2}^{*}\left(1+\rho_{k}^{1}\right)-c \lambda_{1}^{k} \alpha_{1}^{*}\left(1+\rho_{k}^{2}\right)+ \\
& \frac{1}{4 d}\left(b \lambda_{2}^{m}-c \lambda_{1}^{k}\right)^{2}\left(1+\rho_{k}^{3}\right)
\end{aligned}
$$

and a value $\mu=\mu_{p f}^{(k, m)}$, associated to the pitch-fork bifurcation in $T_{k m}$ (which is not conservative if we have in mind Lemma 4),

$$
\begin{aligned}
\mu_{p f}^{(k, m)}= & \lambda_{2}^{m} \alpha_{2}^{*}\left(1+\rho_{k}^{1}\right)-c \lambda_{1}^{k} \alpha_{1}^{*}\left(1+\rho_{k}^{2}\right)- \\
& \frac{3}{4 d}\left(b \lambda_{2}^{m}-c \lambda_{1}^{k}\right)^{2}\left(1+\rho_{k}^{3}\right) .
\end{aligned}
$$

Finally, using formula (3.4) for the curve $P D\left(P_{3,4}\right)$ of simultaneous period doubling bifurcation of the fixed points $P_{3}$ and $P_{4}$ we find that

$$
\begin{aligned}
\mu_{p d}^{(k, m)}= & \lambda_{2}^{m} \alpha_{2}^{*}\left(1+\rho_{k}^{1}\right)-c \lambda_{1}^{k} \alpha_{1}^{*}\left(1+\rho_{k}^{2}\right)- \\
& \frac{1}{4 d}\left(b \lambda_{2}^{m}-3 c \lambda_{1}^{k}\right)\left(3 b \lambda_{2}^{m}-c \lambda_{1}^{k}\right)\left(1+\rho_{k}^{3}\right) .
\end{aligned}
$$

These considerations imply Theorem 1.

\section{On applied reversible maps with mixed dynamics}

In this section we present two concrete examples of reversible differential equations which provide a framework where Theorem 1 applies and exhibiting, therefore, mixed dynamics: a periodically perturbed Duffing equation and the Pikovsky-Topaj model [21] for coupled rotators. 


\subsection{A periodic perturbation of the Duffing Equation}

Let us consider the following system

$$
\left\{\begin{array}{l}
\dot{x}=y \\
\dot{y}=-x+x^{3}+\varepsilon(\alpha+\beta y \sin \omega t)
\end{array}\right.
$$

where $\alpha, \beta, \omega \in \mathbb{R}$ and $\varepsilon$ is an small perturbation parameter. The unperturbed system, for $\varepsilon=0$, corresponds to the so-called Duffing system (also called Anti-Duffing for several authors). It is Hamiltonian, with

$$
H(x, y)=\frac{y^{2}}{2}+V(x), \quad V(x)=\frac{x^{2}}{2}-\frac{x^{4}}{4}-\frac{1}{4}
$$

and is (time)-reversible with respect the following linear involutions $R(x, y)=(x,-y)$ and $S(x, y)=(-x, y)$. This system has three singular points: one elliptic at $(0,0)$ and two saddles at $( \pm 1,0)$. Moreover, these two points are connected through a symmetric couple of heteroclinic orbits $\Gamma_{h}^{-}$.

The perturbed system, for $\varepsilon \neq 0$, is still $R$-reversible but, in principle, non necessarily Hamiltonian. This is a particular case of a more general family of $R$ reversible perturbations

$$
\left\{\begin{array}{l}
\dot{x}=y \\
\dot{y}=-x+x^{3}+\varepsilon g(x, y, t)
\end{array}\right.
$$

satisfying that $g(x,-y,-t)=g(x, y, t)$. It is well known that two (symmetric) hyperbolic periodic orbits $\gamma_{\varepsilon}^{-}$appear close to the saddle points $( \pm 1,0)$. Let us denote by $W^{u, s}\left(\gamma_{\varepsilon}^{-}\right)$their corresponding unstable and stable invariant manifolds, respectively. Generically these invariant manifolds will intersect each other transversally and will remain close to the unperturbed heteroclinic connection. Its splitting is given, at first order in $\varepsilon$, by the celebrated Poincaré-Melnikov-Arnol'd function

$$
M\left(t_{0}\right)=\int_{-\infty}^{+\infty} L_{G} F\left(\Gamma_{h}(t)\right) d t
$$

where $F(x, y)=\left(y,-x+x^{3}\right), G(x, y)=\varepsilon(0, \alpha+\beta y \sin \omega t), \Gamma_{h}(t)$ is any of both unperturbed heteroclinic connections $\Gamma_{h}^{-}(t)$ and where $L_{G}(F)=(D F) G$ corresponds to the Lie derivative of $F$ with respect to $G$. Simple zeroes of $M\left(t_{0}\right)$ provide tangent intersections between the invariant manifolds $W^{u, s}\left(\gamma_{\varepsilon}^{-}\right)$. This systems constitutes a good candidate to apply our results.

For the computation of $M\left(t_{0}\right)$ we consider here the positive heteroclinic orbit $\Gamma_{h}=\Gamma_{h}^{+}$but, by symmetry, everything applies exactly for $\Gamma_{h}^{-}$. Thus,

and

$$
\Gamma_{h}(t)=\left(x_{h}(t), y_{h}(t)\right)=\left(x_{h}(t), \dot{x}_{h}(t)\right)=\left(\tanh \frac{t}{\sqrt{2}}, \frac{1}{\sqrt{2}} \operatorname{sech}^{2} \frac{t}{\sqrt{2}}\right)
$$

$$
\begin{aligned}
& M\left(t_{0}\right)=\left.\int_{-\infty}^{+\infty}(D F) G\right|_{\left(x_{h}(t), y_{h}(t), t+t_{0}\right)} d t= \\
& \int_{-\infty}^{+\infty} y_{h}(t)\left(\alpha+\frac{\beta}{\sqrt{2}}\left(\operatorname{sech}^{2} \frac{t}{\sqrt{2}}\right) \sin \omega\left(t+t_{0}\right)\right) d t= \\
& \frac{\varepsilon \alpha}{\sqrt{2}} \int_{-\infty}^{+\infty} \operatorname{sech}^{2} \frac{t}{\sqrt{2}} d t+\frac{\varepsilon \beta}{2} \int_{-\infty}^{+\infty}\left(\operatorname{sech}^{4} \frac{t}{\sqrt{2}}\right) \sin \omega\left(t+t_{0}\right) d t= \\
& \frac{\varepsilon \alpha}{\sqrt{2}} I_{1}+\frac{\varepsilon \beta}{2} I_{2} .
\end{aligned}
$$


Concerning $I_{1}$ it is straightforward to check that its value is 2 . Regarding $I_{2}$, it is more convenient to compute the integral

$$
\int_{-\infty}^{+\infty}\left(\operatorname{sech}^{4} \frac{t}{\sqrt{2}}\right) \mathrm{e}^{\mathrm{i} \omega\left(t+t_{0}\right)} d t
$$

using the method of residues. Indeed, from it we can derive that

$$
\begin{aligned}
& \int_{-\infty}^{+\infty}\left(\operatorname{sech}^{4} \frac{t}{\sqrt{2}}\right) \sin \omega\left(t+t_{0}\right) d t=\frac{2 \pi}{3} \frac{\omega^{2}\left(\omega^{2}+2\right)}{\sinh \frac{\omega \pi}{\sqrt{2}}} \sin \omega t_{0}, \\
& \int_{-\infty}^{+\infty}\left(\operatorname{sech}^{4} \frac{t}{\sqrt{2}}\right) \cos \omega\left(t+t_{0}\right) d t=\frac{2 \pi}{3} \frac{\omega^{2}\left(\omega^{2}+2\right)}{\sinh \frac{\omega \pi}{\sqrt{2}}} \cos \omega t_{0}
\end{aligned}
$$

and, substituting in (4.3), we get

$$
\begin{aligned}
M\left(t_{0}\right)= & \varepsilon\left(\alpha \sqrt{2}+\frac{\beta \pi}{3} \frac{\omega^{2}\left(\omega^{2}+2\right)}{\sinh \frac{\omega \pi}{\sqrt{2}}} \sin \omega t_{0}\right)= \\
& \frac{\pi \omega^{2}\left(\omega^{2}+2\right)}{3 \sinh \frac{\omega \pi}{\sqrt{2}}} \varepsilon\left(\alpha \mathcal{P}(\omega)+\beta \sin \omega t_{0}\right),
\end{aligned}
$$

provided we define

$$
\mathcal{P}(\omega)=\frac{3 \sqrt{2} \sinh \frac{\omega \pi}{\sqrt{2}}}{\pi \omega^{2}\left(\omega^{2}+2\right)} .
$$

Therefore, for small values of $\varepsilon$ we have: $(i)$ if $|\beta / \alpha|>\mathcal{P}(\omega)$ then $W^{u}\left(\gamma_{\varepsilon}^{-}\right)$and $W^{s}\left(\gamma_{\varepsilon}^{+}\right)$ intersect; $($ ii $)$ if $|\beta / \alpha|<\mathcal{P}(\omega)$ they do not intersect each other and (iii) if $|\beta / \alpha|=\mathcal{P}(\omega)$ then $M\left(t_{0}\right)$ has zeroes which are double but not triple since $\partial M\left(t_{0}\right) / \partial \alpha \neq 0$; this case leads to quadratic heteroclinic tangencies.

\subsection{On the Pikovsky-Topaj model [21] of coupled rotators}

Let us consider the following system

$$
\begin{aligned}
& \dot{\psi}_{1}=1-2 \varepsilon \sin \psi_{1}+\varepsilon \sin \psi_{2} \\
& \dot{\psi}_{2}=1-2 \varepsilon \sin \psi_{2}+\varepsilon \sin \psi_{1}+\varepsilon \sin \psi_{3} \\
& \dot{\psi}_{3}=1-2 \varepsilon \sin \psi_{3}+\varepsilon \sin \psi_{2},
\end{aligned}
$$

where $\psi_{i} \in[0,2 \pi), i=1,2,3$, are cyclic variables. Thus, the phase space of (4.4) is the 3 -dimensional torus $\mathbb{T}^{3}$. System (4.4) is reversible with respect to the involution $\mathcal{R}: \psi_{1} \rightarrow \pi-\psi_{3} \quad, \quad \psi_{2} \rightarrow \pi-\psi_{2} \quad, \quad \psi_{3} \rightarrow \pi-\psi_{1}$.

System (4.4) was suggested by Pikovsky and Topaj in the paper [21] as a simple model describing the dynamics of 4 coupled elementary rotators. By means of the coordinate change

$$
\xi=\frac{\psi_{1}-\psi_{3}}{2}, \quad \eta=\frac{\psi_{1}+\psi_{3}-\pi}{2}, \quad \rho=\frac{\psi_{1}+\psi_{3}-\pi}{2}+\psi_{2}-\pi
$$

and the change in time $d \tau=d t(2+\varepsilon \cos (\rho-\eta))$ system (4.4) is led into

$$
\begin{aligned}
\dot{\xi} & =\frac{2 \varepsilon \sin \xi \sin \eta}{2+\varepsilon \cos (\rho-\eta)} \\
\dot{\eta} & =\frac{1-\varepsilon \cos (\rho-\eta)-2 \varepsilon \cos \xi \cos \eta}{2+\varepsilon \cos (\rho-\eta)} \\
\dot{\rho} & =1
\end{aligned}
$$


Then time-1 Poincaré map of system (4.5) is also reversible with respect to the same involution $R: \quad \xi \rightarrow \xi, \eta \rightarrow-\eta$.

It was found in [21] that, for small $\varepsilon$, system (4.4) behaves itself as a conservative system close to integrable one and several invariant curves could be observed. However, when one increases the value of $\varepsilon$ invariant curves break down and chaos appears (which is already noticed, for instance, at $\varepsilon \approx 0.3$ ). This picture looks to be quite similar to the conservative case. However, certain principal differences take place. In particular, a "strange behavior" of the invariant measure is observed. Iterations of the initial measure are convergent to some suitable limit. However, the limits $t \rightarrow+\infty$ and $t \rightarrow-\infty$ for the same initial measure are different ( numerically observed, for instance, for values of $\varepsilon \approx 0.3$ ). This situation is impossible when the invariant measure is absolutely continuous. Therefore, it must be singular and concentrated on "attractors and conservators" at $t \rightarrow+\infty$ or "on repellers and conservators" at $t \rightarrow-\infty$. Here under the term "conservator" we mean the set of self-symmetric non-wandering orbits. Moreover, $+\infty$ - and $-\infty$-invariant measures look like symmetric (with respect to the fixed line of the involution) and having non-empty intersection so there are no gaps between asymmetric and symmetric parts. This means that "visually" attractors and repellers intersect and it is an evidence of mixed dynamics in this model.

Moreover, a transition from conservative dynamics to non-conservative one can be generated by bifurcations of periodic orbits. For small enough $\varepsilon$ periods of all such orbits are large and the corresponding resonance zones are narrow. When increasing $\varepsilon$, periodic orbits of no too large period appear and dissipative phenomena can become observable. For example, the map $T$ under consideration has no points of period 1 and 2 for $\varepsilon<0.6$ but it has, at $\varepsilon=\varepsilon^{*} \approx 0.445$, two period 3 orbits. Notice that these orbits are different since map $T$ has the symmetry $\xi \rightarrow 2 \pi-\xi$ that implies the appearance of 2 (in fact, an even number) different orbits. Thus, the scenario is the following: there is no fixed point for $T^{3}$ at $\varepsilon<\varepsilon^{*}$; at $\varepsilon=\varepsilon^{*}$ a symmetric couple of fixed points with double multiplier +1 appear in Fix $R$; at $\varepsilon>\varepsilon^{*}$ all these orbits fall into four orbits, a symmetric couple of elliptic orbits and a symmetric couple of contracting-expanding saddle orbits.

Bifurcations of such type are not typical in one-parameter families even in the reversible case. Here, general bifurcations are met (for symmetric fixed points) of types " $0 \rightarrow 2$ " or " $1 \rightarrow 3$ ", that is, "conservative" fold and "reversible" pitchfork, respectively. The presence of a typical bifurcation " $0 \rightarrow 4$ " says us about the existence of a certain additional degeneracy in the system. The "clear symmetry" $\xi \rightarrow-\xi$ is not suitable for this rôle. However, system (4.5) possesses such a "hidden symmetry" which implies that the map $T_{(\rho=0) \rightarrow(\rho=2 \pi)}$ is the second power of some non-orientable map. This peculiarity is caused by the fact that the maps $T_{(\rho=\pi) \rightarrow(\rho=2 \pi)}$ and $T_{(\rho=0) \rightarrow(\rho=\pi)}$ are conjugate. In particular, one can check that

$$
T_{(\rho=\pi) \rightarrow(\rho=2 \pi)}=S^{-1} T_{(\rho=0) \rightarrow(\rho=\pi)} S,
$$

through the linear change of coordinates $\xi \rightarrow \pi-\xi, \eta \rightarrow \eta+\pi, \rho \rightarrow \rho+\pi$. Indeed, after this coordinate transformation, the right sides of system (4.5) remain the same, but the limits of integration (along orbits of system (4.5) to get the correspondence map between sections $\rho=a$ and $\rho=b$ ) are shifted in $\pi$. Such a property is called time-shift symmetry.

From (4.6) it follows that $T_{(\rho=0) \rightarrow(\rho=2 \pi)}=T_{(\rho=0) \rightarrow(\rho=\pi)} S T_{(\rho=0) \rightarrow(\rho=\pi)} S^{-1}$. Since $S^{2}=\mathrm{Id}$, one has that $S=S^{-1}$ and, therefore,

$$
T_{(\rho=0) \rightarrow(\rho=2 \pi)}=\left(T_{(\rho=0) \rightarrow(\rho=\pi)} S\right)^{2}
$$


This means that the map $T_{(\rho=0) \rightarrow(\rho=2 \pi)}$ considered is the second power of some map. Notice that the transformation associated to $S$ is non-orientable and, thus, the map $T_{(\rho=0) \rightarrow(\rho=\pi)} S$ is non-orientable as well and, on its turn, our first-return map $T$ is also the second power of some non-orientable map.

It is straightforward to check that the map $T_{(\rho=0) \rightarrow(\rho=\pi)}$ is reversible with respect to the involution $R_{1}(x, y)=(-x,-y)$ and that the map $T_{(\rho=0) \rightarrow(\rho=\pi)} S$ is reversible under the involution $R(x, y)=(x,-y)$. Thus, the bifurcation of map $T^{3}$ at $\varepsilon=\varepsilon^{*}$ can be treated as a bifurcation of a fixed point with multipliers $(+1,-1)$ in the case of a non-orientable map (in fact, the map $\left.\left(T_{(\rho=0) \rightarrow(\rho=\pi)} S\right)^{3}\right)$. So, summarising, in our case this bifurcation leads to the appearance of two elliptic points of period 2 on Fix $R$ and a symmetric couple of saddle fixed points (that is, outside Fix $R$ and symmetric one to each other). These saddle fixed points are not conservative. It can be checked numerically that the Jacobian of one point is greater than 1 and less than 1 at other point. Due to reversibility, the stable and unstable manifolds of saddles pairwise intersect and form a "heteroclinic tangle" zone. This zone is extremely narrow since the separatrix splitting is exponentially small. However, moving slightly away from the bifurcation moment we can find numerically heteroclinic tangencies and, hence, moments of creation of non-transversal heteroclinic cycles. Since the saddles involved are not conservative, it follows from [15] the phenomenon of mixed dynamics.

\section{Cross-form type equations for reversible maps. Proof of Lemmas 1 and 2}

\subsection{Cross-form for reversible maps}

As it will be seen along this section, the so-called Shilnikov cross-form variables constitute a very useful way to deal with reversible maps and a simple way to generate them. We say that a map is in cross-form if it is written as

$$
\left\{\begin{array}{l}
\bar{x}=h_{1}(x, \bar{y}), \\
y=h_{2}(x, \bar{y}),
\end{array}\right.
$$

where the regularity of $h_{1,2}$ varies depending on the problem. In this paper we will assume $\left(h_{1}, h_{2}\right)$ to give rise to a $C^{r}$ diffeomorphism.

On the other hand, let us consider a diffeomorphism $F$ of the plane which is reversible with respect to a (in general, non-linear) involution $R\left(R^{2}=\mathrm{id}, R \neq \mathrm{id}\right)$, with the curve of points fixed under $R$ of dimension 1 . Remind that this means that $F \circ R \circ F=R$ - or, equivalently, $R \circ F \circ R=F^{-1}$ since $F$ is a diffeo - and $\operatorname{dim} \operatorname{Fix} R=1$. Assume that the involution $R$ reverses orientation, which is the most common situation in the literature. Our aim is to show that cross-form type can be very suitable when dealing with reversible planar maps.

We start considering the simplest setting, that is, when the reversor $R$ is the linear (orientation-reversing) involution $L:(x, y) \mapsto(y, x)$. In this case, the following result holds:

Lemma 6 Any diffeomorphism $F:(x, y) \mapsto(\bar{x}, \bar{y})$ defined, implicitly, by means of equations of type

$$
F:\left\{\begin{array}{l}
\bar{x}=f(x, \bar{y}) \\
y=f(\bar{y}, x)
\end{array}\right.
$$

is always reversible with respect to $L(x, y)=(y, x)$. 
Proof. Since $F$ is a $L$-reversible diffeomorphism it satisfies that $L \circ F \circ L=F^{-1}$ or, equivalently, $(L \circ F \circ L)^{-1}=F$, which is the expression we will prove at the end. To do it, we first need a convenient equivalent expression for the inverse of a planar diffeomorphism. Indeed, if

$$
H:\left\{\begin{array}{l}
\bar{x}=h_{1}(x, y), \\
\bar{y}=h_{2}(x, y),
\end{array}\right.
$$

the corresponding inverse map $H^{-1}:(x, y) \mapsto(\bar{x}, \bar{y})$ can be implicitly written through the expression

$$
H^{-1}:\left\{\begin{array}{l}
x=h_{1}(\bar{x}, \bar{y}) \\
y=h_{2}(\bar{x}, \bar{y})
\end{array}\right.
$$

This is clear since $(x, y)=H(\bar{x}, \bar{y})$ implies that $(\bar{x}, \bar{y})=H^{-1}(x, y)$. An algorithmic way to get it consists just on swapping bars among the variables, that is $x \leftrightarrow \bar{x}$ and $y \leftrightarrow \bar{y}$. We apply this procedure to compute formally an expression for $(L \circ F \circ L)^{-1}$ and to check afterwards that it coincides with $F$. Let us do it step by step. First we have

$$
F \circ L:\left\{\begin{array}{l}
\bar{x}=f(y, \bar{y}) \\
x=f(\bar{y}, y)
\end{array}\right.
$$

To apply $L$ onto $F \circ L$ corresponds to swap $\bar{x} \leftrightarrow \bar{y}$ in the precedent expression:

$$
L \circ F \circ L:\left\{\begin{array}{l}
\bar{y}=f(y, \bar{x}), \\
x=f(\bar{x}, y) .
\end{array}\right.
$$

And finally, to get its inverse we swap bars and no-bars, that is, $x \leftrightarrow \bar{x}$ and $y \leftrightarrow \bar{y}$. Performing this change we obtain

$$
(L \circ F \circ L)^{-1}:\left\{\begin{array}{l}
y=f(\bar{y}, x), \\
\bar{x}=f(x, \bar{y}),
\end{array}\right.
$$

which is exactly the expression for $F$.

This result can be useful to provide suitable local expressions for planar reversible diffeomorphisms. Namely, we have the following lemma.

Lemma 7 Let $F=\left(f_{1}, f_{2}\right)$ be a planar diffeomorphism, reversible with respect a general involution $R, \mathcal{C}^{r}, r \geq 1$, orientation reversing and with $\operatorname{dim} \operatorname{Fix} R=1$. Let us assume the origin $(0,0)$ a fixed point of the involution $R$, that is $(0,0) \in \operatorname{Fix} R$.

Then, if $D_{x x} f_{1}+D_{y y} f_{2} \neq 0$ at $(0,0)$ there exist local coordinates, that we denote again by $(x, y)$, in which $F$ admits the following implicit (normal) form

$$
\left\{\begin{array}{l}
\bar{x}=g(x, \bar{y}), \\
y=g(\bar{y}, x) .
\end{array}\right.
$$

This map is reversible with respect to $L(x, y)=(y, x)$.

In the case of a saddle fixed point, the concrete type of implicit normal form that can be obtained is given in equation (3.3).

Proof. It will be proved in two steps:

(i) First we apply Bochner Theorem [2] which allows us to conjugate, around $(0,0)$, our involution $R$ to its linear part $\left.D R\right|_{(0,0)}$.

(ii) Using that the partial derivatives on $(0,0)$ do not vanish simultaneously, we apply Implicit Function Theorem to reach the final form. 
We proceed as follows:

(i) Notice that if $R$ is a (general) involution and $p \in$ Fix $R$ then its linear part $\left.D R\right|_{p}$ is an involution as well. Indeed,

$$
\mathrm{Id}=R^{2} \Rightarrow I=D\left(R^{2}\right)(p)=\left.\left.D R\right|_{R(p)} \cdot D R\right|_{p}=\left(\left.D R\right|_{p}\right)^{2} .
$$

Bochner Theorem ensures the existence of a $\mathcal{C}^{r}$-diffeo $\psi$ which conjugates, locally around $p, R$ to $\left.D R\right|_{p}$. We include, for completeness, a simple proof of this fact given in [23]. From the equality

$$
\begin{array}{r}
\left.D R\right|_{p} \circ\left(R+\left.D R\right|_{p}\right)=\left.D R\right|_{p} \circ R+\mathrm{id}= \\
\quad \mathrm{id}+\left.D R\right|_{p} \circ R=\left(R+\left.D R\right|_{p}\right) \circ R
\end{array}
$$

it follows that $\left.D R\right|_{p} \circ\left(R+\left.D R\right|_{p}\right)=\left(R+\left.D R\right|_{p}\right) \circ R$. We define $\psi=R+\left.D R\right|_{p} \in \mathcal{C}^{r}$ and check that it is a diffeomorphism in a neighbourhood of $p$ :

$$
\left.D \psi\right|_{p}=\left.D\left(R+\left.D R\right|_{p}\right)\right|_{p}=\left.D R\right|_{p}+\left.D R\right|_{p}=\left.2 D R\right|_{p}
$$

and so det $\left.D \psi\right|_{p}=\left.2 \operatorname{det} D R\right|_{p} \neq 0$ since $R$ is a diffeomorphism around $p$. So $\psi$ is a $\mathcal{C}^{r}$-diffeomorphism which conjugates $R$ to $\left.D R\right|_{p}$ around $p$.

Since $R$ is orientation reversing its linear part around $p,\left.D R\right|_{p}$, is also orientation reversing. Following [23] for instance, we know that there exists a transformation which conjugates $\left.D R\right|_{p}$ to the linear involution $L(x, y)=(y, x)$, which will be the one we will consider, locally, from now on.

(ii) Let us assume, for instance, that $D_{y y} f \neq 0$ at $(0,0)$. Using Implicit Function Theorem, we can write from equation $\bar{y}=f_{2}(x, y)$ an expression for $y$, say $y=g(\bar{y}, x)$, for a suitable function $g$. Substituting it into the equations defining $F$ we get a (locally) equivalent expression for $F$ :

$$
F:\left\{\begin{array}{l}
\bar{x}=f_{1}(x, y)=f_{1}(x, g(\bar{y}, x))=: h(x, \bar{y}) \\
y=g(\bar{y}, x)
\end{array}\right.
$$

As stated above, we can assume $F$ to be locally conjugated around the origin to the linear involution $L:(x, y) \mapsto(y, x)$. So in that variables (to simplify the notation we keep the same name for the variables and the functions involved) it must satisfy that $(L \circ F \circ L)^{-1}=F$. Applying the procedure introduced in Lemma 6 , one obtains that

$$
F \circ L:\left\{\begin{array}{l}
\bar{x}=h(y, \bar{y}) \\
x=g(\bar{y}, y) .
\end{array}\right.
$$

We apply $L$ (that corresponds to swapping $\bar{x}$ and $\bar{y}$,

$$
L \circ F \circ L:\left\{\begin{array}{l}
\bar{y}=h(y, \bar{x}) \\
x=g(\bar{x}, y)
\end{array}\right.
$$

and, finally, we swap $(x, y)$ for $(\bar{x}, \bar{y})$,

$$
(L \circ F \circ L)^{-1}:\left\{\begin{array}{l}
y=h(\bar{y}, x), \\
\bar{x}=g(x, \bar{y}) .
\end{array}\right.
$$

Since it must coincide with $F$ it turns out that $h(x, \bar{y})=g(x, \bar{y})$ and so

$$
F:\left\{\begin{array}{l}
\bar{x}=g(x, \bar{y}) \\
y=g(\bar{y}, x)
\end{array}\right.
$$

We present now a counterpart result when the map is given in implicit form. 
Lemma 8 Any map $G:(x, y) \mapsto(\bar{x}, \bar{y})$ given by

$$
\left\{\begin{array}{l}
g(x, y, \bar{x}, \bar{y})=0 \\
g(\bar{y}, \bar{x}, y, x)=0
\end{array}\right.
$$

is $L$-reversible, where $L:(x, y) \mapsto(y, x)$. The second equation $g(\bar{y}, \bar{x}, y, x)=0$ is " $a$ kind of" L-conjugate of the first equation $g(x, y, \bar{x}, \bar{y})=0$.

Proof. It is enough to check that $L \circ G \circ L=G^{-1}$. To do it we proceed again as in Lemma 6. First, remind that an implicit expression for $G^{-1}$ is always obtained by swapping bars for no-bars, that is, $(x, y) \leftrightarrow(\bar{x}, \bar{y})$. So

$$
G^{-1}:\left\{\begin{array}{l}
g(\bar{x}, \bar{y}, x, y)=0 \\
g(y, x, \bar{y}, \bar{x})=0
\end{array}\right.
$$

On the other hand we compute $L \circ G \circ L$. Thus,

$$
G \circ L:\left\{\begin{array}{l}
g(y, x, \bar{x}, \bar{y})=0 \\
g(\bar{y}, \bar{x}, x, y)=0
\end{array}\right.
$$

and, swapping $(\bar{x}, \bar{y})$ for $(\bar{y}, \bar{x})$, we get

$$
L \circ G \circ L:\left\{\begin{array}{l}
g(y, x, \bar{y}, \bar{y})=0, \\
g(\bar{x}, \bar{y}, x, y)=0,
\end{array}\right.
$$

which coincides with $G^{-1}$. Therefore the lemma is proved.

The following result establishes an interesting relation between polynomial reversible and area preserving maps.

Lemma 9 ([22]) Any Taylor truncation of a planar polynomial diffeomeorphism which is reversible with respect to a linear involution is area preserving. In particular, this applies to the truncation of a normal form of such diffeomorphisms.

Proof. Let $\bar{z}=G(z)$ a polynomial planar map which is reversible with respect to a linear involution $S\left(S^{2}=\mathrm{Id}, S \neq \mathrm{Id}\right)$. This means that $S \circ G \circ S=G^{-1}$ and, in particular, that $G^{-1}$ is also a polynomial. Differentiating the latter expression we get

$$
\begin{aligned}
& \left.S D G\right|_{S z} S=\left.D\left(G^{-1}\right)\right|_{z}=\left(\left.D G\right|_{G^{-1}(z)}\right)^{-1} \Rightarrow \\
& \operatorname{det}\left(\left.S D G\right|_{S z} S\right)=\frac{1}{\left.\operatorname{det} D G\right|_{G^{-1}(z)}} .
\end{aligned}
$$

Using that $\operatorname{det}\left(\left.S D G\right|_{S z} S\right)=\left.(\operatorname{det} S)^{2} \operatorname{det} D G\right|_{S z}=\left.\operatorname{det} D G\right|_{S z}$ it follows that

$$
\left(\left.\operatorname{det} D G\right|_{S z}\right) \cdot\left(\left.\operatorname{det} D G\right|_{G^{-1}(z)}\right)=1, \quad \forall z \text {. }
$$

Since $G$ and $G^{-1}$ are polynomials and $S$ linear we obtain that $\left.\operatorname{det} D G\right|_{S z}$ and $\left.\operatorname{det} D G\right|_{G^{-1}(z)}$ are polynomials as well. But the product of two polynomials is a constant if and only if they are constant, that is, $\left.\operatorname{det} D G\right|_{z} \equiv k=$ constant. Thus, from (5.2) it follows that $k^{2}=1$ and, therefore, $\left.\operatorname{det} D G\right|_{z}= \pm 1, \forall z$.

And last, but not least, we remark another interesting property regarding this cross-form type: any polynomial truncation of a reversible diffeomorphism written in cross-form type is also in cross-form type and, consequently, it is reversible. This means, from Lemma 9, that this truncation is always area-preserving. 


\subsection{Proof of Lemma 1}

Let $O$ be a fixed saddle point of a reversible map $T_{0}$. Applying Bochner Theorem [16], we can assume the existence of local coordinates around $O$ such that $O$ is located at the origin and that the involution $R$ is exactly $(x, y) \mapsto(y, x)$ in these coordinates.

Let $x=\nu(y)$ be the equation of the stable manifold. Then, by the $R$-reversibility, $y=\nu(x)$ is the equation of the unstable manifold. If $|d \nu / d y|<1$, we perform the transformation $x_{n e w}=x-\nu(y), y_{\text {new }}=y-\nu(x)$, while, if $|d \nu / d y|>1$, the change is $x_{n e w}=y-\nu(x), y_{n e w}=x-\nu(y)$. After such transformation, which commutes with $R$, the equations of the stable and unstable manifolds become $y=0$ and $x=0$, respectively. Thus, in the corresponding local coordinates, the map can be represented in the following form

$$
\bar{x}=\lambda x+g_{1}(x, y), \quad \bar{y}=\lambda^{-1} y+g_{2}(x, y)
$$

where $g_{1}(0, y) \equiv 0, g_{2}(x, 0) \equiv 0$ and $g_{i}^{\prime}(0,0)=0, i=1,2$. It is very convenient to rewrite this equation in the so-called cross-form:

$$
\bar{x}=\lambda x+\tilde{g}_{1}(x, \bar{y}), \quad y=\lambda \bar{y}+\tilde{g}_{2}(x, \bar{y})
$$

Equation (5.4) comes from (5.3) writing $y=F(x, \bar{y})$ (which exists due to the Implicit Function Theorem) and substituting it into the first equation: $\bar{x}=\lambda x+g_{1}(x, F(x, \bar{y}))$. The $R$-reversibility of (5.4) implies that $\tilde{g}_{1}(x, y) \equiv \tilde{g}_{2}(y, x)$ so we can represent map (5.4) in the form

$$
\begin{aligned}
& \bar{x}=\lambda x+\varphi_{1}(x)+\psi_{1}(\bar{y}) x+\rho_{1}(x, \bar{y}) x^{2} \bar{y} \\
& y=\lambda \bar{y}+\varphi_{1}(\bar{y})+\psi_{1}(x) \bar{y}+\rho_{1}(\bar{y}, x) x \bar{y}^{2}
\end{aligned}
$$

Performing the $R$-invariant change of variables

$$
\xi=x+x h_{1}(y), \quad \eta=y+y h_{1}(x)
$$

with $h_{1}(0)=0$, it turns out the following equation for $\bar{\xi}$ :

$$
\begin{aligned}
\bar{\xi}= & \bar{x}+\bar{x} h_{1}(\bar{y})= \\
& \lambda \xi-\lambda x h_{1}(y)+x \psi_{1}(\bar{y})+\left(\lambda x+\psi_{1}(\bar{y}) x+\varphi_{1}(x)\right) h_{1}(\bar{y})+ \\
& \varphi_{1}(\xi)+O\left(\xi^{2} \bar{\eta}\right)= \\
& \lambda \xi+\varphi_{1}(\xi)+O\left(\xi^{2} \bar{y}\right)+ \\
& x\left[-\lambda h_{1}\left(\lambda \bar{y}+\varphi_{1}(\bar{y})\right)+\psi_{1}(\bar{y})+\left(\lambda+\psi_{1}(\bar{y})\right) h_{1}(\bar{y})\right] .
\end{aligned}
$$

Since we want the expression in the square brackets to vanish identically, we ask the function $h_{1}(y)$ to satisfy the functional equation

$$
h_{1}\left(\lambda \bar{y}+\varphi_{1}(\bar{y})\right)=h_{1}(\bar{y})\left(1+\lambda^{-1} \psi_{1}(\bar{y})\right)+\lambda^{-1} \psi_{1}(\bar{y}),
$$

which has solutions $h_{1}=h_{1}(u)$ in the class of $C^{r-1}$-functions. Indeed, we can consider (5.7) as an equation for the strong stable invariant manifold of the following planar map

$$
\begin{aligned}
\bar{h}_{1}(\bar{u}) & =\left(1+\lambda^{-1} \psi_{1}(u)\right) h_{1}(u)+\lambda^{-1} \psi_{1}(u), \\
\bar{u} & =\lambda u+\varphi_{1}(u) .
\end{aligned}
$$

Since $0<|\lambda|<1, \psi_{1}(0)=0$ and $\varphi_{1}(0)=\varphi_{1}^{\prime}(0)=0$, this map has strong stable invariant manifold $W^{s s}$ passing through the origin, that is, satisfying an equation $h_{1}=h_{1}(u)$ with $h_{1}(0)=0$. Therefore, after the $R$-invariant change (5.6), the map (5.5) takes the form

$$
\begin{aligned}
& \bar{x}=\lambda x+\varphi_{1}(x)+\rho_{2}(x, \bar{y}) x^{2} \bar{y} \\
& y=\lambda \bar{y}+\varphi_{1}(\bar{y})+\rho_{2}(\bar{y}, x) x \bar{y}^{2}
\end{aligned}
$$


Applying a $R$-invariant change of variables of the form

$$
\xi=x+h_{2}(x) x, \quad \eta=y+h_{2}(y) y
$$

with $h_{2}(0)=0$, the first equation of system (5.8) can be rewritten, in these new coordinates, as follows

$$
\begin{aligned}
\bar{\xi}= & \lambda \xi+x\left[-\lambda h_{2}(x)+\tilde{\varphi}_{1}(x)+\right. \\
& \left.h_{2}\left(\lambda x+\varphi_{1}(x)\right)\left(\lambda+\tilde{\varphi}_{1}(x)\right)\right]+O\left(\xi^{2} \eta\right),
\end{aligned}
$$

where we have denoted $\varphi_{1}(x) \equiv \tilde{\varphi}_{1}(x) x$. As we did above for $h_{1}$, we seek for a function $h_{2}$ satisfying the following equation

$$
h_{2}(\lambda x+\varphi(x))=\left(1+\lambda^{-1} \tilde{\varphi}_{1}(x)\right)^{-1}\left(h_{2}(x)-\lambda^{-1} \tilde{\varphi}_{1}(x)\right),
$$

which vanishes the expression inside the square brackets in (5.9). As before, equation (5.10) has solutions $h_{2}=h_{2}(u)$ in the class of $C^{r-1}$-functions. Again, one can consider the expression (5.10) as an equation for the strong stable invariant manifold associated to the following planar map

$$
\begin{aligned}
& \bar{h}_{2}=\left(1+\lambda^{-1} \tilde{\varphi}_{1}(x)\right)^{-1}\left(h_{2}-\lambda^{-1} \tilde{\varphi}_{1}(x)\right), \\
& \bar{u}=\lambda u+\varphi_{1}(u) .
\end{aligned}
$$

Having in mind that $0<|\lambda|<1$ and $\varphi_{1}(0)=\varphi_{1}^{\prime}(0)=0$, this map admits strong stable invariant manifold $W^{s s}$ passing through the origin, i.e., having an equation $h_{2}=h_{2}(u)$ with $h_{2}(0)=0$. This completes the proof of the Lemma.

\subsection{Proof of Lemma 2}

We write the map $T_{0}$ in the following form

$$
\bar{x}=\lambda x+\hat{h}(x, y), \quad \bar{y}=\gamma y+\hat{g}(x, y)
$$

where we assume that $\gamma=\lambda^{-1}$ and

$$
\hat{h}(x, y) \equiv x^{2} y\left(\beta_{1}+O(|x|+|y|)\right), \quad \hat{g}(x, y) \equiv x y^{2}\left(\beta_{2}+O(|x|+|y|)\right) .
$$

Consider the following operator $\Phi:\left[\left(x_{j}, y_{j}\right)\right]_{j=0}^{k} \mapsto\left[\left(\bar{x}_{j}, \bar{y}_{j}\right)\right]_{j=0}^{k}$ :

$$
\begin{aligned}
& \bar{x}_{j}=\lambda^{j} x_{0}+\sum_{s=0}^{j-1} \lambda^{j-s-1} \hat{h}\left(x_{s}, y_{s}, \mu\right), \\
& \bar{y}_{j}=\gamma^{j-k} y_{k}-\sum_{s=j}^{k-1} \gamma^{j-s-1} \hat{g}\left(x_{s}, y_{s}, \mu\right),
\end{aligned}
$$

where $j=0,1, \ldots, k$. The operator $\Phi$ is defined on the set

$$
Z(\delta)=\left\{z=\left[\left(x_{j}, y_{j}\right)\right]_{j=0}^{k},\|z\| \leq \delta\right\},
$$

where the norm $\|\cdot\|$ is given as the maximum of modulus of components $x_{j}, y_{j}$ of the vector $z$. Notice that if $z_{0}=\left[\left(x_{j}^{0}, y_{j}^{0}\right)\right]_{j=0}^{k}$ is a fixed point of $\Phi$, then the following diagram takes place

$$
\left(x_{0}^{0}, y_{0}^{0}\right) \stackrel{T_{0}}{\longrightarrow}\left(x_{1}^{0}, y_{1}^{0}\right) \stackrel{T_{0}}{\longrightarrow} \ldots \stackrel{T_{0}}{\longrightarrow}\left(x_{k}^{0}, y_{k}^{0}\right),
$$

i.e. the fixed point of $\Phi$ gives a segment of an orbit of $T_{0}$.

It is known [1] that, for small enough $\delta=\delta_{0}$ and $\left|x_{0}\right| \leq \delta_{0} / 2,\left|y_{k}\right| \leq \delta_{0} / 2$, the operator $\Phi$ maps the set $Z\left(\delta_{0}\right)$ into itself and it is contracting. Thus, map (5.11) has a unique fixed point $z_{0}=\left[\left(x_{j}^{0}\left(x_{0}, y_{k}\right), y_{j}^{0}\left(x_{0}, y_{k}\right)\right]_{j=0}^{k}\right.$ that is limit of iterations under $\Phi$ for any initial point from $Z\left(\delta_{0}\right)$. Thus, the coordinates $x_{j}^{0}$ and $y_{j}^{0}$ can be found by 
applying successive approximations. As an initial approximation, we take the solution of the linear problem:

$$
x_{j}^{0(1)}=\lambda^{j} x_{0}, \quad y_{j}^{0(1)}=\gamma^{j-k} y_{k}
$$

It follows from (5.11) that the second approximation has a form

$$
\begin{aligned}
& x_{j}^{0(2)}=\lambda^{j} x_{0}+\sum_{s=0}^{j-1} \lambda^{j-s-1} \lambda^{2 s} \gamma^{s-k} x_{0}^{2} y_{k} \times \\
& \left(\beta_{1}+O\left(|\lambda|^{s}\left|x_{0}\right|+|\gamma|^{s-k}\left|y_{k}\right|\right)\right)= \\
& \lambda^{j} x_{0}+\lambda^{j} \gamma^{-k} \sum_{s=0}^{j-1} \lambda^{-1} \lambda^{s} \gamma^{s} x_{0}^{2} y_{k}\left(\beta_{1}+O\left(|\lambda|^{s}\left|x_{0}\right|+|\gamma|^{s-k}\left|y_{k}\right|\right)\right)= \\
& \lambda^{j} x_{0}+(j-1) \lambda^{j} \gamma^{-k} \lambda^{-1} x_{0}^{2} y_{k}\left(\beta_{1}+O\left(|\lambda|^{s}\left|x_{0}\right|+|\gamma|^{s-k}\left|y_{k}\right|\right)\right), \\
& y_{j}^{0(2)}=\gamma^{j-k} y_{k}+\sum_{s=j}^{k-1} \gamma^{j-s-1} \lambda^{s} \gamma^{2(s-k)} x_{0} y_{k}^{2} \times \\
& \left(\beta_{2}+O\left(|\lambda|^{s}\left|x_{0}\right|+|\gamma|^{s-k}\left|y_{k}\right|\right)\right)= \\
& \gamma^{j-k} y_{k}+\gamma^{j-2 k} \sum_{s=j}^{k-1} \gamma^{-1} \lambda^{s} \gamma^{s} x_{0} y_{k}^{2}\left(\beta_{2}+O\left(|\lambda|^{s}\left|x_{0}\right|+|\gamma|^{s-k}\left|y_{k}\right|\right)\right)= \\
& \gamma^{j-k} y_{k}+(k-j) \gamma^{j-2 k-1} x_{0} y_{k}^{2}\left(\beta_{2}+O\left(|\lambda|^{s}\left|x_{0}\right|+|\gamma|^{s-k}\left|y_{k}\right|\right)\right)
\end{aligned}
$$

Since $\gamma=\lambda^{-1}$, it follows from the precedent expression that

$$
\begin{aligned}
& \left|x_{j}^{0(2)}-\lambda^{j} x_{0}\right| \leq L_{1} j \lambda^{j+k} \\
& \left|y_{j}^{0(2)}-\lambda^{k-j} y_{k}\right| \leq L_{2}(k-j) \lambda^{2 k-j}
\end{aligned}
$$

where $L_{1}$ and $L_{2}$ are some positive constant independent of $j$ and $k$. Substituting (5.12) into (5.11) as the initial approximation, then the following ones will also satisfy estimates (5.12), with the same constants $L_{1}$ and $L_{2}$. Thus, formula (3.5) is valid for the coordinates $x_{l}^{0}$ and $y_{0}^{0}$ of the fixed point of $\Phi$.

The estimates for the derivatives of the functions $x_{l}^{0}$ and $y_{0}^{0}$ are deduced in the same way as done in [8] (see also modified versions of the proof in $[27,13,14]$ ).

\section{Proofs of Lemmas 3, 4 and 5 .}

\subsection{Proof of Lemma 3}

Since coordinates $\left(x_{01}, y_{01}\right)$ on $\sigma_{k}^{01}$ are uniquely determined via cross-coordinates $\left(x_{01}, y_{11}\right)$ in equations $(3.6)$, we can express $T_{k m}$ as a map defined on points $\left(x_{01}, y_{11}\right)$ and acting by the rule $\left(x_{01}, y_{11}\right) \mapsto\left(\bar{x}_{01}, \bar{y}_{11}\right)$. As a result of this, we can express the map $T_{k m}$ in the following form

$$
\begin{aligned}
& x_{02}-x_{2}^{+}=a \lambda_{1}^{k} x_{01}+b\left(y_{11}-y_{1}^{-}\right)+l_{02}\left(y_{11}-y_{1}^{-}\right)^{2}+ \\
& \tilde{\varphi}_{1 k}\left(x_{01}, y_{11}-y_{1}^{-}, \mu\right), \\
& \lambda_{2}^{m} y_{12}\left(1+m \lambda_{2}^{m} h_{m}^{2}\left(y_{12}, x_{02}, \mu\right)\right)=\mu+c \lambda_{1}^{k} x_{01}+d\left(y_{11}-y_{1}^{-}\right)^{2}+ \\
& f_{11} \lambda_{1}^{k} x_{01}\left(y_{11}-y_{1}^{-}\right)+f_{03}\left(y_{11}-y_{1}^{-}\right)^{3}+\tilde{\varphi}_{2 k}\left(x_{01}, y_{11}-y_{1}^{-}, \mu\right), \\
& \lambda_{2}^{m} x_{02}\left(1+m \lambda_{2}^{m} h_{m}^{2}\left(x_{02}, y_{12}, \mu\right)\right)=\mu+c \lambda_{1}^{k} \bar{y}_{11}+d\left(\bar{x}_{01}-y_{1}^{-}\right)^{2}+ \\
& \quad f_{11} \lambda_{1}^{k} \bar{y}_{11}\left(\bar{x}_{01}-y_{1}^{-}\right)+f_{03}\left(\bar{x}_{01}-y_{1}^{-}\right)^{3}+\tilde{\varphi}_{2 k}\left(y_{11}-y_{1}^{-}, x_{01}, \mu\right), \\
& y_{12}-x_{2}^{+}=a \lambda_{1}^{k} \bar{y}_{11}+b\left(\bar{x}_{01}-y_{1}^{-}\right)+l_{02}\left(\bar{x}_{01}-y_{1}^{-}\right)^{2}+ \\
& \tilde{\varphi}_{1 k}\left(y_{11}-y_{1}^{-}, x_{01}, \mu\right),
\end{aligned}
$$


where the coordinates $x_{02}$ and $y_{12}$ are "intermediate" and

$$
\begin{aligned}
& \tilde{\varphi}_{1 k}(u, v, \mu)=O\left(\lambda_{1}^{2 k} u^{2}+\left|\lambda_{1}^{k}\right||u v|+|v|^{3}\right), \\
& \tilde{\varphi}_{2 k}(u, v, \mu)=O\left(\lambda_{1}^{2 k}\left(u^{2}+|u| v^{2}\right)+\left|\lambda_{1}^{k}\right||u| v^{2}\right)+o\left(v^{3}\right) .
\end{aligned}
$$

Now we perform the following shift in the coordinates

$$
\begin{array}{ll}
\xi_{1}=x_{01}-x_{1}^{+}+\nu_{k m}^{1}, & \eta_{1}=y_{11}-y_{1}^{-}+\nu_{k m}^{1}, \\
\xi_{2}=x_{02}-x_{2}^{+}+\nu_{k m}^{2}, & \eta_{2}=y_{12}-y_{2}^{-}+\nu_{k m}^{2},
\end{array}
$$

where $\nu_{k m}^{i}=O\left(\lambda_{1}^{k}\right), i=1,2$, are some small coefficients which does not destroy the reversibility due to the condition (3.5). Then, for suitable $\nu_{k m}^{i}$, map $T_{k m}$ becomes

$$
\begin{aligned}
\xi_{2}= & a \lambda_{1}^{k} \xi_{1}+b \eta_{1}+l_{02} \eta_{1}^{2}+\tilde{\varphi}_{1 k}\left(\xi_{1}, \eta_{1}, \mu\right), \\
\lambda_{2}^{m} \eta_{2} & \left(1+m \lambda_{2}^{m} h_{m}^{2}\left(\eta_{2}, \xi_{2}, \mu\right)\right)= \\
& \tilde{\mu}+c \lambda_{1}^{k} \xi_{1}+d \eta_{1}^{2}+f_{11} \lambda_{1}^{k} \xi_{1} \eta_{1}+f_{03} \eta_{1}^{3}+\tilde{\varphi}_{2 k}\left(\xi_{1}, \eta_{1}, \mu\right), \\
\lambda_{2}^{m} \xi_{2} & \left(1+m \lambda_{2}^{m} h_{m}^{2}\left(\xi_{2}, \eta_{2}, \mu\right)\right)= \\
& \tilde{\mu}+c \lambda_{1}^{k} \bar{\eta}_{1}+d \bar{\xi}_{1}^{2} f_{11} \lambda_{1}^{k} \bar{\eta}_{1} \bar{\xi}_{1}+f_{03} \bar{\xi}_{1}^{3}+\tilde{\varphi}_{2 k}\left(\bar{\eta}_{1}, \bar{\xi}_{1}, \mu\right), \\
\eta_{2}= & a \lambda_{1}^{k} \bar{\eta}_{1}+b \bar{\xi}_{1}+l_{02} \bar{\xi}_{1}^{2}+\tilde{\varphi}_{1 k}\left(\bar{\eta}_{1}, \bar{\xi}_{1}, \mu\right),
\end{aligned}
$$

where, since relation (3.5) holds, we have that $\tilde{\mu}=\mu-\lambda_{2}^{m}\left(\alpha_{2}^{*}+\ldots\right)+c \lambda_{1}^{k}\left(\alpha_{1}^{*}+\ldots\right)$ and the new functions $\tilde{\varphi}_{1 k}$ and $\tilde{\varphi}_{2 k}$ satisfy again conditions (6.1). One must, however, to consider coefficients $a, b, \ldots, f_{03}$ in $(6.2)$ to be shifted by values of order $O\left(k \lambda_{1}^{k}\right)$ when comparing them with the initial coefficients in (3.7). Substituting into the second and third equations of (6.2) the expressions for $\xi_{2}$ and $\eta_{2}$ given by the first and the fourth precedent equations, we get an expression for $T_{k m}$ of form

$$
\begin{aligned}
& \lambda_{2}^{m}\left(a \lambda_{1}^{k} \bar{\eta}_{1}+b \bar{\xi}_{1}+l_{02} \bar{\xi}_{1}^{2}\right)= \\
& \tilde{\mu}+c \lambda_{1}^{k} \xi_{1}+d \eta_{1}^{2}+f_{11} \lambda_{1}^{k} \xi_{1} \eta_{1}+f_{03} \eta_{1}^{3}+\tilde{\varphi}_{2 k}, \\
& \lambda_{2}^{m}\left(a \lambda_{1}^{k} \xi_{1}+b \eta_{1}+l_{02} \eta_{1}^{2}\right)= \\
& \tilde{\mu}+c \lambda_{1}^{k} \bar{\eta}_{1}+d \bar{\xi}_{1}^{2}+f_{11} \lambda_{1}^{k} \bar{\eta}_{1} \bar{\xi}_{1}+f_{03} \bar{\xi}_{1}^{3}+\tilde{\varphi}_{2 k},
\end{aligned}
$$

which can be rewritten as

$$
\begin{aligned}
& a \lambda_{2}^{m} \bar{\eta}_{1}+b \lambda_{2}^{m} \lambda_{1}^{-k} \bar{\xi}_{1}+l_{02} \lambda_{2}^{m} \lambda_{1}^{-k} \bar{\xi}_{1}^{2}= \\
& \tilde{\mu} \lambda_{1}^{-k}+c \xi_{1}+d \lambda_{1}^{-k} \eta_{1}^{2}+f_{11} \xi_{1} \eta_{1}+f_{03} \lambda_{1}^{-k} \eta_{1}^{3}+\lambda_{1}^{-k} \tilde{\varphi}_{2 k}, \\
& a \lambda_{2}^{m} \xi_{1}+b \lambda_{2}^{m} \lambda_{1}^{-k} \eta_{1}+l_{02} \lambda_{2}^{m} \lambda_{1}^{-k} \eta_{1}^{2}= \\
& \tilde{\mu} \lambda_{1}^{-k}+c \bar{\eta}_{1}+d \lambda_{1}^{-k} \bar{\xi}_{1}^{2}+f_{11} \bar{\eta}_{1} \bar{\xi}_{1}+f_{03} \lambda_{1}^{-k} \bar{\xi}_{1}^{3}+\lambda_{1}^{-k} \tilde{\varphi}_{2 k} .
\end{aligned}
$$

Even thought they are represented by the same letter, the functions $\tilde{\varphi}_{2 k}$ in the latter expression may be different of those in (6.2). We just want to stress that they still fulfil relations (6.1). Finally, rescaling coordinates,

$$
\xi_{1}=\lambda_{1}^{k} x, \quad \eta_{1}=\lambda_{1}^{k} y,
$$

system (6.3) takes the form (3.10) where the coefficients $c, d, \ldots, l_{02}$ are the "original" ones (that is, those appearing in formula (3.7)).

\subsection{Proof of Lemma 4}

The rescaled form (3.10) of the first-return map $T_{k m}$ is, of course, implicit one and it corresponds to a formal representation $T_{k m}: F(\bar{x}, \bar{y}) \equiv G(x, y)$ which can be written 
in the explicit form $(\bar{x}, \bar{y}) \equiv T_{k m}(x, y) \equiv F^{-1} G(x, y)$. Then we can find the Jacobian of $T_{k m}$ using the relation

$$
\left.\left.D\left(T_{k m}\right)\right|_{(x, y)} \equiv D\left(F^{-1}\right)\right|_{G(x, y)} D(G(x, y)),
$$

where $D(\cdot)$ is the corresponding (differential) Jacobi matrix. At the fixed point $\left(x=\xi^{*}, y=\eta^{*}\right)$ of $T_{k m}$ we can rewrite (6.4) as follows

$$
\left.\left.D\left(T_{k m}\right)\right|_{\left(\xi^{*}, \eta^{*}\right)} \equiv\left(\left.D F\right|_{\left(\xi^{*}, \eta^{*}\right)}\right)^{-1} D G\right|_{\left(\xi^{*}, \eta^{*}\right)} .
$$

We find from (3.10) that $D F$ and $D G$ are of the form

$$
\begin{aligned}
& D F=\left(\begin{array}{cc}
2 d \xi^{*}+f_{11} \eta^{*} \lambda_{1}^{k}+3 f_{03} \lambda_{1}^{k}\left(\xi^{*}\right)^{2} & c+f_{11} \xi^{*} \lambda_{1}^{k} \\
b \lambda_{1}^{-k} \lambda_{2}^{m}+2 \lambda_{2}^{m} l_{02} \xi^{*} & a \lambda_{2}^{m}
\end{array}\right), \\
& D G=\left(\begin{array}{cc}
a \lambda_{2}^{m} & b \lambda_{1}^{-k} \lambda_{2}^{m}+2 \lambda_{2}^{m} l_{02} \eta^{*} \\
c+f_{11} \eta^{*} \lambda_{1}^{k} & 2 d \eta^{*}+f_{11} \xi^{*} \lambda_{1}^{k}+3 \lambda_{1}^{k} f_{03}\left(\eta^{*}\right)^{2}
\end{array}\right)
\end{aligned}
$$

plus terms of order $O\left(k \lambda_{1}^{2 k}\right)$. Now we compute the Jacobian as

$$
\begin{aligned}
& \left.J\left(T_{k m}\right)\right|_{\left(\xi^{*}, \eta^{*}\right)}=\operatorname{det}\left((D F)^{-1} D G\right)=\frac{\operatorname{det}(D G)}{\operatorname{det}(D F)}= \\
& \frac{-b c \lambda_{1}^{-k} \lambda_{2}^{m}+2 a d \lambda_{2}^{m} \eta^{*}-b f_{11} \lambda_{2}^{m} \eta^{*}-2 c l_{02} \lambda_{2}^{m} \eta^{*}+o\left(\lambda_{1}^{k}\right)}{-b c \lambda_{1}^{-k} \lambda_{2}^{m}+2 a d \lambda_{1}^{k} \lambda_{2}^{m} \xi^{*}-b f_{11} \lambda_{2}^{m} \xi^{*}-2 c l_{02} \lambda_{2}^{m} \xi^{*}+o\left(\lambda_{1}^{k}\right)} .
\end{aligned}
$$

When the relation (2.2) is fulfilled we can rewrite (6.5) as

$$
\left.J\left(T_{k m}\right)\right|_{\left(\xi^{*}, \eta^{*}\right)}=\frac{-b c+Q \lambda_{1}^{k} \eta^{*}+o\left(\lambda_{1}^{k}\right)}{-b c+Q \lambda_{1}^{k} \xi^{*}+o\left(\lambda_{1}^{k}\right)}
$$

that gives relation $(3.19)$.

\subsection{Proof of Lemma 5}

It is not restrictive to prove Lemma 5 for the truncated map $H$, written in explicit form as

$$
H:\left\{\begin{aligned}
\bar{x} & =\tilde{M}+\tilde{c} x-y^{2}, \\
\bar{y} & =-\frac{1}{\tilde{c}} \tilde{M}+\frac{1}{\tilde{c}} y+\frac{1}{\tilde{c}} \bar{x}^{2} .
\end{aligned}\right.
$$

We know that for $M>-\frac{1}{4}(\tilde{c}-1)^{2}$ it has a pair of symmetric fixed points $P^{+}=\left(p_{+}, p_{+}\right)$ and $P^{-}=\left(p_{-}, p_{-}\right)$given by formula (3.17). Denote by $p$ either $p_{+}$or $p_{-}$and let us assume that the corresponding fixed point $P$ (i.e. $P^{+}$or $P^{-}$) is elliptic. Then, $\tilde{c}$ and $\tilde{M}$ have to take values from the open regions in the $(\tilde{c}, \tilde{M})$-space of parameters given in Figure 8.

The first step in our process is to shift the new origin of coordinates to this point $P=(p, p)$ and to perform (Jordan) linear normal form, which leads our map to the form

$$
\begin{aligned}
\bar{x}=\cos \psi & \cdot x-\sin \psi \cdot y-\frac{2 p \cos \psi}{\tilde{c} \sin \psi} y^{2}+ \\
& +\frac{1-4 p^{2}-\tilde{c} \cos \psi}{4 \tilde{c}^{2} p^{2} \sin \psi}\left(-\tilde{c} \sin \psi \cdot x+(1-\tilde{c} \cos \psi) y+2 p y^{2}\right)^{2} \\
\bar{y}=\sin \psi & \cdot x+\cos \psi \cdot y-\frac{2 p}{\tilde{c}} y^{2}+ \\
& +\frac{1}{4 \tilde{c} p^{2}}\left(-\tilde{c} \sin \psi \cdot x+(1-\tilde{c} \cos \psi) y+2 p y^{2}\right)^{2}
\end{aligned}
$$


where $x, y, \bar{x}, \bar{y}$ stand again for the new variables. The linear part of (6.6) is a rotation of angle $\psi$. Now we lead our map into Birkhoff Normal Form up to order 3, namely: $\bar{z}=\mathrm{e}^{\mathrm{i} \psi} z+d_{21} z^{2} z^{*}+\mathcal{O}_{4}$. To do it, we need to assume that $\lambda=\mathrm{e}^{\mathrm{i} \psi}$ is not a $k$ th-root of unity for $k=3,4$ (the cases $k=1,2$ correspond to parabolic fixed points and, therefore, to boundaries of existence regions of elliptic fixed points in the $(\tilde{c}, \tilde{M})$-parameter space). The coefficient $B_{1} \equiv-i d_{21} e^{-i \psi}$ is called the first Birkhoff coefficient. By the Arnol'd-Moser Twist Theorem [28], the inequality $B_{1} \neq 0$ (together with the absence of strong resonances) ensures that the elliptic point is generic or, in other words, KAM-stable.

Introducing complex coordinates $z=x+\mathrm{i} y, z^{*}=x-\mathrm{i} y$, map (6.6) takes the form

$$
\bar{z}=\mathrm{e}^{\mathrm{i} \psi} z+A_{20} z^{2}+A_{11} z z^{*}+A_{02}\left(z^{*}\right)^{2}+A_{21} z^{2} z^{*}+\mathcal{O}_{4}\left(z, z^{*}\right) .
$$

Since we are assuming $\mathrm{e}^{\mathrm{i} \psi}$ not to be a 3 rd or 4 th root of unity (and also $\psi \neq 0, \pi$ ), our map can be lead into BNF up to order 3 and, afterwards, provides the following formula for the first Birkhoff coefficient

$$
B_{1}=\frac{(\tilde{c}+1-2 p)(\tilde{c}+1+2 p)(\tilde{c}-1+2 p)}{32 \tilde{c}^{4} p \sin ^{3} \psi(2 \cos \psi+1)} Q_{4}(\tilde{c}, p),
$$

where

$$
\begin{gathered}
Q_{4}(\tilde{c}, p)=64 p^{4}+8(1-\tilde{c}) p^{3}-4\left(3 \tilde{c}^{2}+4 \tilde{c}+3\right) p^{2}+ \\
2(\tilde{c}-1)(\tilde{c}+1)^{2} p-(\tilde{c}-1)^{2}(\tilde{c}+1)^{2} .
\end{gathered}
$$

Using this formula\| we represent in Figure 9 curves $B_{1}(\tilde{c}, \tilde{M})=0$, where the elliptic fixed point can be, a priori, not KAM-stable. In this Figure curves related to the strong resonances are presented. For resonances $1: 1$ and $1: 2$ the equations of the corresponding curves are given in Section 3.4. The equations of the 1:3 and 1:4 curves are as follows:

$$
\tilde{M}=\frac{\tilde{c}^{2}+1}{4} \pm \frac{\sqrt{\tilde{c}^{2}+1}}{2}(1-\tilde{c}) \quad \text { for } 1: 4 \text { resonance }
$$

and

$$
\tilde{M}=\frac{\tilde{c}^{2}+\tilde{c}+1}{4} \pm \frac{\sqrt{\tilde{c}^{2}+\tilde{c}+1}}{2}(1-\tilde{c}) \quad \text { for } 1: 3 \text { resonance. }
$$

This completes the proof.

\section{Conclusion}

Generally speaking, mixed dynamics phenomenon should be considered as one more, new, kind of chaotic dynamics distinct in many principal properties from strange attractors and conservative chaos. As it follows from the work of D.Turaev [30], mixed dynamics can be observed in any dimension (in particular, Newhouse domain with mixed dynamics should exist near specific non-transversal heteroclinic cycles

$\|$ Notice that in the particular case $\tilde{c}=-1$, map $H$ corresponds to $\mathcal{H}^{2}$, where $\mathcal{H}: \bar{x}=y, \bar{y}=$ $M-x-y^{2}$ is the Hénon map. In this case we have that $B_{1}^{H}(\psi)=2 B_{1}^{\mathcal{H}(\varphi)}$ where $\psi=2 \varphi$. It is not hard to check now that, for a fixed point of $\mathcal{H}$ with $p=-\cos \varphi$, the following relation holds

$$
B_{1}^{\mathcal{H}^{2}}=B_{1}^{\mathcal{H}^{2}}=\frac{1}{4 \sin ^{2} \varphi} \cdot \frac{(1+\cos \varphi)(1+4 \cos \varphi)}{\sin \varphi(1+2 \cos \varphi)},
$$

which differs from the well-known formula for the Hénon map (see e.g. [3]) only by the non-zero factor $\frac{1}{4} \sin ^{-2} \varphi$. 


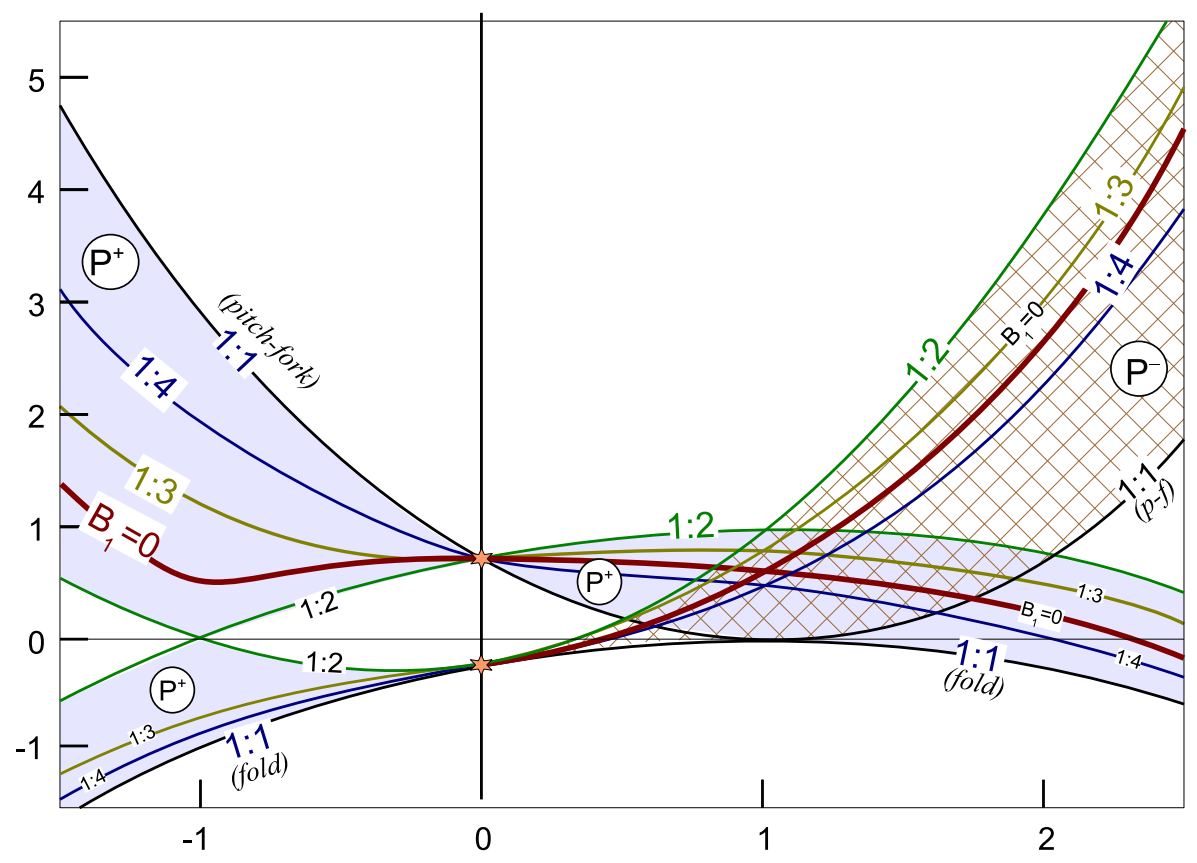

Figure 9. In the $(\tilde{c}, \tilde{M})$-plane, three grey and one hatching regions correspond to the existence of elliptic points: $P^{+}$for the grey regions and $P^{-}$for the hatching one. Lines corresponding to the main resonances and vanishing the first Birkhoff coefficient for the elliptic point are shown and labelled.

proposed in [30]). However, the corresponding problem looks to be very complicated and requires quite delicate consideration.

Regarding the two dimensional reversible case, a kind of systematic program could be followed to prove the RMD-conjecture in the one parameter context and for the main cases of diffeomorphisms with symmetric homoclinic or heteroclinic tangencies.

In Figure 10 we collect some simple examples of such maps. They differ by the type of fixed points and tangencies: homoclinic or heteroclinic, quadratic or cubic $\mathbf{\uparrow}$, etc. It seems to be more important its division into two groups: a first one including those maps which are a priori non-conservative and a second one with those maps where this non-conservativity is, in some sense, hidden.

A map as in Figure 1(a) (the first one in Figure 10), containing a symmetric couple of contracting-expanding saddles, belongs to the first group since condition $J\left(O_{1,2}\right) \neq 1$ destroys certainly the conservative character. Indeed, under splitting such heteroclinic cycle, homoclinic tangencies appear both to saddles with Jacobian

I The existence of symmetric cubic homoclinic or heteroclinic tangencies is a codimension one bifurcation phenomenon in the class of reversible maps. Therefore, these cubic tangencies should be also considered as the main ones jointly with the pointed out quadratic ones. 


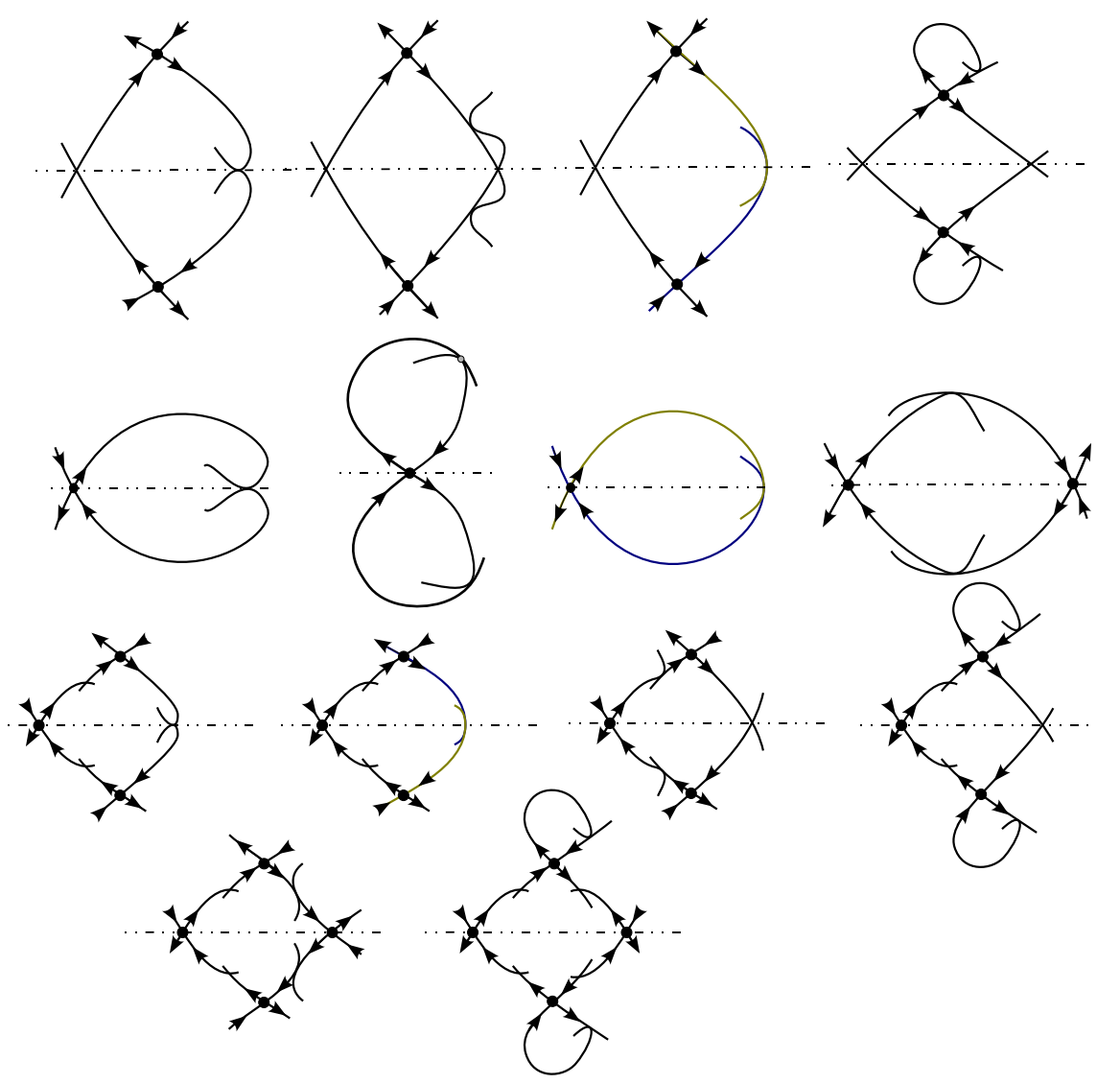

Figure 10. Some examples of reversible maps with homoclinic and heteroclinic tangencies

greater and less than 1 and, thus, attracting and repelling periodic points can be born [6]. By the same argument, all the other cases with a symmetric couple of contractingexpanding fixed (periodic) points are a priori non-conservative maps (in particular, all maps at the first, third and fourth lines in Figure 10). For maps of this type, the problem of finding symmetric elliptic periodic orbits should be considered of special relevance.

The maps at the second line of Figure 10 have only symmetric fixed points. The first, third and fourth maps can be considered as maps with "hidden nonconservativity" since, in principle, it is not clear the existence of bifurcation mechanisms leading to the appearance of attracting and repelling periodic orbits.

This is not the case for the second map (again at the second line) which has a symmetric couple of (quadratic) homoclinic tangencies to the same symmetric fixed saddle point. As in the first map at the first line, one can assume without loss of reversibility that the first-return map near a homoclinic point is not conservative, giving rise to a symmetric couple of contracting-expanding homoclinic orbits. As for 
the "a priori conservative case", the main problem to be considered is to prove the appearance of elliptic periodic orbits and, as a first step, to do it in the one-parameter setting.

Acknowledgements. The authors thank D. Turaev and J.S.W. Lamb for fruitful discussions and remarks. They are also indebted to the referees for many suggestions and comments on a first version of this paper. The second and fifth authors have been supported partially by the Russian Federation Government grant, contract No.11.G34.31.0039 and grants of RFBR No.10-01-00429, 11-01-00001 and 11-01-97017 r-povoljie. The first and fourth authors have been partially supported by the MICIIN/FEDER grant number MTM2009-06973 and by the Generalitat de Catalunya grant number 2009SGR859. The second, third and fourth authors also thank Centre de Recerca Matemàtica (Bellaterra) for its hospitality during a semester in 2008 .

[1] Afraimovich V S and Shilnikov L P, 1973, On critical sets of Morse-Smale systems Trans. Moscow Math. Soc. 28, 179-212.

[2] Bochner S., 1945, Compact groups of differentiable transformations. Ann. Math., second series, vol. 46, 3, 372-381.

[3] Biragov V.S. "Bifurcations in a two-parameter family of conservative mappings that are close to the Henon map".- Selecta Math.Sov., 1990, v.9, 273-282. [Originally publ. in "Methods of qualitative theory of differential equations", Gorky State Univ., 1987, 10-24.]

[4] Delshams A and Lázaro J T, 2005, Pseudo-normal form near saddle-center or saddle-focus equilibria J. Differential Equations 208(2) 312-343.

[5] Duarte P, 2000, Persistent homoclinic tangencies for conservative maps near the identity Ergod. Th. Dyn. Sys. 20 393-438.

[6] Gavrilov N K and Shilnikov L P, 1972, On three-dimensional dynamical systems close to systems with a structurally unstable homoclinic curve I, Math. USSR Sbornik, 17, 467-485; II, 1973, 19, 139-156.

[7] Gonchenko S V and Shilnikov L P, 1990, Invariants of $\Omega$-conjugacy of diffeomorphisms with a structurally unstable homoclinic trajectory Ukrainian Math. J. 42 134-140.

[8] Gonchenko S V and Shilnikov L P, 1993, Moduli of systems with a structurally unstable homoclinic Poincaré curve Russian Acad. Sci. Izv. Math., 41(3) 417-445.

[9] Gonchenko S V, Turaev D V and Shilnikov L P, 1993, On the existence of Newhouse regions near systems with non-rough Poincaré homoclinic curve (multidimensional case) Russian Acad. Sci.Dokl.Math., $\mathbf{4 7 .}$

[10] Gonchenko S V, Turaev D V and Shilnikov L P, 1997, On Newhouse domains of two-dimensional diffeomorphisms with a structurally unstable heteroclinic cycle Proc. Steklov Inst. Math., 216, $70-118$.

[11] Gonchenko S V and Shilnikov L P, 2000, On two-dimensional area-preserving diffeomorphisms with infinitely many elliptic islands J.of Stat.Phys. 101(1/2), 321-356.

[12] Gonchenko S V and Gonchenko V S, 2004, On bifurcations of birth of closed invariant curves in the case of two-dimensional diffeomorphisms with homoclinic tangencies Proc. Steklov Inst. 244, 80-105.

[13] Gonchenko S V, Shilnikov L P and Turaev D, 2007, Homoclinic tangencies of arbitrarily high orders in conservative and dissipative two-dimensional maps Nonlinearity 20, 241-275.

[14] Gonchenko S V, Shilnikov L P and Turaev D, 2008, On dynamical properties of multidimensional diffeomorphisms from Newhouse regions Nonlinearity 21(5), 923-972.

[15] Lamb J S W and Stenkin O V, 2004, Newhouse regions for reversible systems with infinitely many stable, unstable and elliptic periodic orbits Nonlinearity 17(4), 1217-1244.

[16] Montgomery D and Zippin L 1955, Topological transformation groups Intersience, New York.

[17] Newhouse, S E, 1970, Non-density of Axiom A(a) on $S^{2}$, Proc. A.M.S. Symp. Pure Math., 14, pp.191-202.

[18] Newhouse S, 1974, Diffeomorphisms with infinitely many sinks Topology 13 9-18.

[19] Newhouse S E, 1979, The abundance of wild hyperbolic sets and non-smooth stable sets for diffeomorphisms Publ. Math. Inst. Hautes Etudes Sci. 50 101-151. 
[20] Palis J and Viana M, 1994, High dimension diffeomorphisms displaying infinitely many sinks Ann. Math. 140 91-136.

[21] Pikovsky A, Topaj D, 2002, "Reversibility vs. synchronization in oscillator latties", Physica D, v.170, 118-130.

[22] Roberts, J A G , 1997, Some characterisations of low-dimensional dynamical systems with timereversal symmetry. Math. Model. 8, Control \& Chaos.

[23] Roberts J A G and Quispel G R W , 1992, Chaos and time-reversal symmetry. Order and Chaos in reversible systems. Physics Reports, 216, Numbers 2-3, pages 63-177.

[24] Romero N, 1995, Persistence of homoclinic tangencies in higher dimensions Ergod. Th. Dyn.Sys. 15 735-757.

[25] Shilnikov L P, 1967, On a Poincaré-Birkhoff problem Math. USSR Sb. 3 91-102.

[26] Shilnikov L P, 1977, The bifurcation theory of dynamical systems with Poincaré homoclinic curves, 1977, in book: VII Internationale Konferenz über nichtlineare Schwingungen, Band 1, 2, Academie Verlag, Berlin, 279-293.

[27] Shilnikov L P, Shilnikov A L, Turaev D V and Chua L O 1998, Methods of qualitative theory in nonlinear dynamics. Part I (Singapore: World Scientific).

[28] Siegel CC L and Moser J K, 1995, Lectures on Celestial Mechanics (Berlin, Heidelberg, New York: Springer-Verlag).

[29] Tedeschini-Lalli L and Yorke J A, 1986 How often do simple dynamical processes have infinitely many coexisting sinks? Commun.Math.Phys. 106, 635-657.

[30] Turaev D V, 1996, On dimension of nonlocal bifurcational problems Int. J. of Bifurcation and Chaos 6(5), 919-948.

[31] Turaev D, 2003, Polynomial approximations of symplectic dynamics and richness of chaos in non-hyperbolic area-preserving maps Nonlinearity 16, 123-135.

[32] Yorke J A and Alligood K T, 1983, Cascades of period-doubling bifurcations: a prerequisite for horseshoes, Bull. Amer. Math. Soc. 9, 319-322. 